Erschienen in: Kallmeyer, Werner (Hrsg.): Kommunikation in der Stadt. Teil 1.

Exemplarische Analyse des Sprachverhaltens in Mannheim. Berlin/New York: de Gruyter, 1994. S. 250-317. (Schriften des Instituts für Deutsche Sprache 4 (4.1))

\title{
4. Formelhaftes Sprechen in der Filsbachwelt
}

WERNER KALLMEYER / INKEN KEIM

1. Zielsetzung

2. Zur Forschung über formelhaftes Sprechen

3. Formulierungsverfahren für Charakterisierungsformeln

3.1. Relevanzsetzung und Zuspitzung

3.2. Kondensierung und Gestaltschließung

3.3. Kommentarformat

3.4. Entindexikalisierung $\quad 267$

3.5. Generalisierung 268

3.6. Metaphorisierung 268

3.7. Interaktionsmodalität der fraglosen Sicherheit 269

3.8. Abgrenzung gegen andere Äußerungstypen 271

3.8.1. Andere kondensierte Charakterisierungen 271

3.8.2. Andere Züge $\quad 272$

3.8.3. Themeneinführungen 273

4. Formelhaftes Sprechen im Tratsch: „Der arme Otto” 274

4.1. Themeneinführung und kondensierte erste Behandlung 275

4.2. Konkretisierung und Detaillierung durch eine Erzählung 282

4.3. Thematische Ausweitung 285

4.4. Abschließende Bewertung 287

4.5. Zusätze, Belege, Problematisierungsversuche 288

4.6. Zusammenfassung 295

5. Spiel und Witz 296

6. Soziale Abgrenzung durch formelhaftes Sprechen 301

7. Fazit: „Mach kä schbrisch” 308

8. Beispieltext: „Der arme Otto” 309 


\section{Zielsetzung}

In diesem Beitrag konzentrieren wir uns auf das formelhafte Sprechen und seine Bedeutung für die Symbolisierung der sozialen Identität. 'Formelhaftes Sprechen' benutzen wir als Oberbegriff für den gesamten Bereich der verfestigten Formulierungen von den Phraseologismen im engeren Sinne (Lexemverbindungen) bis hin zu Redensarten, Sprichwörtern, Sentenzen und Routineformeln. ${ }^{1}$ Im Unterschied zu vielen Arbeiten zur Phraseologie, welche formelhafte Ausdrücke in bezug auf ihre Integration in das Sprachsystem, insbesondere also ihre Lexikalisierung betrachten, untersuchen wir die Formelhaftigkeit primär unter dem Aspekt der Sprachverwendung, d.h. als Formelhaftigkeit des Sprechens. Im Vordergrund steht dabei der Zusammenhang zwischen

- Formulierungsverfahren für formelhaftes Sprechen, d.h. für die Herstellung von formelhaften Ausdrücken und die Markierung des Sprechens als formelhaft,

- Regeln des Sprechens, insbesondere den Thematisierungsregeln sowie den Regeln für unterschiedliche Interaktionsmodalitäten (Ernst, Scherz usw.)

- und der sozialen Organisation des Wissens, d.h. dem Aufbau von Wissensbeständen, ihrer Weitergabe und Bearbeitung.

Das Material stammt - wie schon im vorausgehenden Beitrag - aus der mehrjährigen Beobachtung einer Gruppe älterer Frauen aus der Westlichen Unterstadt. Die beobachteten Filsbacherinnen haben ein reiches Repertoire an formelhaften Ausdrücken. Im Material erscheinen praktisch alle bekannten Spielarten von Formeln. Dazu gehören u. a. verschiedene Spruchformen, d.h. Formeln von Satzformat wie:

- wo kän kläger is is kän rischter

- wer net alt werde will soll sich jung hänge

- demm wu=s so gemach werd demm gherd=s ned onnerschd („wer sich so etwas gefallen läßt, ist selber schuld")

- robb emol em e fedder raus die er net hot („reiß mal einem eine Feder aus, die er nicht hat")

1 Die vorliegenden Klassifikationen sind unterschiedlich fein. Ein generell verwendetes Kriterium ist die Unterscheidung zwischen Lexemverbindungen und satzwertigen Phraseologismen. Eine relativ reiche, wenn auch nicht sehr systematische Klassifikation ist die „struktursemantische Mischklassifikation” von Burger et al. (1982, S. 30/31): sie enthält

1. Phraseologische Ganzheiten

2. Phraseologische Verbindungen und bevorzugte Analysen

3. Modellbildungen

4. Phraseologische Vergleiche

5. Streckformen des Verbs

6. Zwillingsformeln

7. Phrase Jlogische Termini

8. Feste Phrasen

9. Sprichwörter und Gemein plätze. 
- reiß e hoar raus wu känni sin

- jedem das seine

- laß blumen sprechen

- beim fresse lernd ma die leid kenne

- versenk des ding ('etwas verschwinden lassen')

Phraseologismen im engeren Sinne sind:

- nichts zu melden haben

- mattscheibe haben

- einen stich ins grüne haben

- $s$ brot net üwwer nacht hawwe ('arm sein', 'sein Geld schwer verdienen')

- uff de hewwel saufe ( auf den Hebel saufen”, d.h. 'in der Kneipe anschreiben lassen').

Die verwendeten Formeln sind in unterschiedlichem Grade spezifisch in der Verbreitung und in ihrer Verbindung zu den Wissensbeständen und dem Erfahrungshintergrund der Filsbachwelt. Neben Formeln, die Allgemeingut in der Gegenwartsgesellschaft sind, wie nichts zu melden haben oder wo kein Kläger ist, ist kein Richter gibt es solche, die eine gewisse Milieu- oder Weltspezifik haben, weil sie einen Erfahrungshintergrund voraussetzen, der milieuspezifisch ist, d.h. nicht filsbachspezifisch im geographischen, wohl aber im sozialen Sinne:

- uff de hewwel saufe

- die milsch frißt mid aus de schissel („die knappen Vorräte verbrauchen sich sehr schnell").

Der Erfahrungshintergrund ist hier die traditionelle Armut eines großen Teils der Filsbachbevölkerung sowie Kneipenbesuche mit Alkoholkonsum als zentralem Inhalt der Freizeit. Daneben gibt es Formeln mit lokal-geographischem Bezug wie versenk des ding im Hafen, der an die Filsbach angrenzt, oder leid die iwwer=s gaswerk zahle als Charakterisierung armer Leute, die etwas in sehr kleinen Raten abzahlen; der historische Ursprung ist, daB vor dem Krieg das Gaswerk an die umliegende innenstädtische Bevölkerung Rundfunkgeräte (Volksempfänger) gegen eine minimale Rate von monatlich zwei Mark abgegeben hat.

Es gibt auch Fälle, in denen eine derartige Formelbildung unter Bezug auf Anlässe in der lokalen Welt als gegenwärtiger ProzeB zu beobachten ist. Ein Beispiel dafür ist die Maxime mehl hieschidde ('sich mit einfachen Mitteln zu helfen wissen'). Der konkrete Hintergrund dieser Formel ist, daB eine der Filsbachfrauen, die sich darüber ärgerte, daB die Hunde immer vor ihren Eingang pinkelten, Mehl ausstreute, das für die Hundebesitzer dem an vielen Stellen im Viertel gestreuten Rattengift zum Verwechseln ähnlich sah; diese führten ihre Hunde daraufhin an andere Stellen. Diese Geschichte ist viel erzählt und zu einem Karnevalsvortrag verarbeitet worden. Eingeweihte, welche die Geschichte kennen, verwenden die Maxime jetzt auch in übertragener Bedeutung. 
Schließlich gibt es auch in der Gruppeninteraktion geprägte Formeln, die $\mathrm{zwar}$ allgemeineren rhetorischen Mustern folgen, die aber in ihrer inhaltlichen Füllung und in der Verwendung an die Interaktionsgeschichte der Gruppe gebunden sind. Sehr häufig werden Formeln zur Charakterisierung beim Reden über Dritte verwendet. Ein sehr ergiebiges Thema ist ein allen Beteiligten bekanntes originelles Ehepaar, Gerda und Otto. Bei der Behandlung dieses Themas erscheinen häufig Charakterisierungsformeln wie:

- der krigg=de eisschrongg abgschlosse

- er kriggd nudle mid schinke sie frißd zwee schdeeks

- er gehd hääm mach=die bedde * sie gehd in die lokale zum schnorre.

Diese Art des formelhaften Sprechens zeichnet sich einmal durch inhaltliche Stereotypie aus. Die Stereotypie wird von den Beteiligten interaktiv durch die Art der Thematisierung aufgezeigt, die eindeutig selbstverständliche Bekanntheit signalisiert (für AuBenstehende ist das zum Teil nicht ohne weiteres transparent). Charakteristisch sind weiter bestimmte Eigenschaften der Formulierungsweise wie Generalisierung bzw. Verabsolutierung von Eigenschaften, Geschlossenheit der Formulierung, Kürze, prägnante Antithesen (zwischen „ihm” und „ihr”) usw. Allerdings sind die Übergänge zu anderen, nicht formelhaften Redeweisen fließend. Oft sind es vor allem Eigenschaften der Sprechweise, welche die Formelhaftigkeit signalisieren. So sind z.B. Sequenzen des formelhaften Sprechens, in denen mehrere solcher Charakterisierungen aufeinanderfolgen, im Kontext aufgrund ihrer prosodischen Merkmale sehr auffällig.

An diesen Charakterisierungsformeln lassen sich aktuelle Prozesse der Formelbildung aufzeigen. Unsere Materialien aus der Bastelgruppe eignen sich sehr gut für solche Analysen. Die Gruppeninteraktionen sind zentrale soziale Ereignisse für die Beteiligten; die Beteiligten haben ein konstantes Themenpotential, und wir konnten die Gruppeninteraktion über mehrere Jahre beobachten. Insofern lassen sich Prozesse der Wiederholung und Reformulierung nicht nur im aktuellen Gesprächszusammenhang verfolgen, sondern auch im historischen GruppenprozeB.

Außerdem ist bei den im Gruppenkontext gebildeten Formeln der Kontextbezug, d.h. die Verbindung zu speziellen Wissensbeständen besonders gut rekonstruierbar, weil diese Wissensbestände im Laufe der Interaktionsgeschichte der Gruppe thematisiert werden. Die gruppenspezifischen Formeln sind in besonderer Weise eigene Sprache. Die Wahl derartiger Formulierungen hat dementsprechend eine große Bedeutung für die Selbst- und Fremddarstellung. Im Vergleich mit diesen Formulierungen werden dann auch die Verwendungsregeln und Kontextbezüge für andere Arten formelhaften Sprechens besser deutlich.

Im folgenden wollen wir nach einem kurzen Forschungsüberblick (2.) einige Verfahren zur Bildung von Charakterisierungsformel zusammenstellen (3.) und deren Einsatz im Interaktionszusammenhang an einem komplexeren Beispiel untersuchen (4.). Dann werden wir noch einmal gesondert auf zwei Aspekte der 
Formelverwendung eingehen, und zwar auf den Zusammenhang von Interaktionsmodalitäten und Formelhaftigkeit am Fall von Phantasiespiel und Witz (5.) sowie auf Formen der sozialen Abgrenzung durch formelhaftes Sprechen (6.).

\section{Zur Forschung über formelhaftes Sprechen}

Insgesamt ist die Phraseologieforschung im Hinblick auf die Lexikologie, aber auch für den Bereich der Spruchformen und Routineformeln relativ weit fortgeschritten. Für die Behandlung der Phraseologie in lexikologischer Hinsicht liegen eine ganze Reihe von Arbeiten vor (vgl. z. B. Fleischer 1982, Gréciano 1983), und Gréciano urteilt sogar: „Die strukturelle Beschreibung der deutschen Phraseologie kann inzwischen als abgeschlossen gelten" (1983, S. 234). Für die Spruchformen gibt es neben Sammlungen wie denen von Röhrich (z.B. 1973) und einigen interessanten volkskundlichen Arbeiten (z.B. Hain 1951; Groeber-Glück 1974; Röhrich/Mieder 1977) auch einige linguistische Arbeiten (vgl. Gülich 1978; Burger/Buhofer/Sialm 1982), und auch für Routineformeln liegen erste Arbeiten vor (vgl. z.B. Coulmas 1981a). In diesen Arbeiten wird auch der Stellenwert der Phraseologie für die linguistische Theoriebildung diskutiert. Für den lexikologischen Bereich spielt z.B. die Frage eine zentrale Rolle, inwieweit die Phraseologismen - in Analogie zur Wortbildung - als modelliert angesehen werden können, d.h., inwieweit die Vielzahl unterschiedlicher Phraseologismen durch Rekurs auf allgemeinere Bildungsmuster erklärt werden kann (vgl. z.B. Fleischer 1982; Gréciano 1983). Für die Bezüge zur Grammatik weist Coulmas (1981a) in Anlehnung an Chafe (1968) darauf hin, daB idiomatische Ausdrücke, da sie zum normalen Bestand jeder Sprache gehören, als Prüfstein für die Angemessenheit von Grammatiktheorien anzusehen seien. Allgemeinere Überlegungen zur Pragmatik sind in diesem Zusammenhang noch selten (vgl. allerdings Coulmas 1981a sowie die vorliegenden Arbeiten zu sprachlichen Stereotypen wie Quasthoff 1973, Wenzel 1978 und Quasthoff 1983). Das liegt u.a. daran, daß viele der für eine Betrachtung der Phraseologie aus der Perspektive der Sprachverwendung wichtigen Gesichtspunkte in der vorliegenden Forschung nur in Hinweisen angelegt, aber kaum ausgearbeitet sind. ${ }^{2}$

Die Soziolinguistik des formelhaften Sprechens ist trotz einiger programmatischer Darstellungen weitgehend unterentwickelt. Auf das sozio- und ethnolinguistische Erkenntnispotential der Untersuchung von sprachlichen Stereotypen ist verschiedentlich hingewiesen worden (z.B. Coulmas 1979 und 1981a; Quasthoff 1983), aber insgesamt sind die damit zusammenhängenden Fragen bislang eher in der Volkskunde als in der Soziolinguistik behandelt worden. Im soziolinguistischen Kontext gibt es Anknüpfungspunkte insbesondere im Zusam-

2 Vgl. z.B. Burger et al. (1982), Kapitel 4.2.2 „Gruppensprachen” und Kapitel 6.4.3 „Der Gebrauch von Phraseologismen”. Einzubeziehen wäre auch die Forschung zur rituellen Kommunikation, die z.T. āhnliche Phānomene untersucht; vgl. u.a. Lüger (1983), Werlen (1984), Paul (1990). 
menhang mit der Stereotypen-Forschung (so bei Bernstein 1959), in der Gruppensprachenforschung (vgl. z.B. Henne 1986) und in den Analysen von Gumperz zur Kontextualisierung (1982). Im folgenden soll kein neuer Forschungsbericht zur Phraseologie geliefert werden, sondern nur eine kurze Betrachtung der Anknüpfungspunkte, die uns für eine Behandlung des formelhaften Sprechens aus soziolinguistischer Perspektive wichtig erscheinen.

(a) Formelhaftigkeit des Sprechens und Definitionskriterien für Phraseologismen

Für unser Vorgehen bei der Analyse, das grundsätzlich von der Materialbeobachtung ausgeht, ist die Frage der Erkennbarkeit des formelhaften Sprechens vielfach ein schwieriges Problem. Man kann zu seiner Lösung zunächst einmal von den phraseologischen Definitionskriterien ausgehen. Die drei entscheidenden und allgemein anerkannten Kriterien sind „Mehrgliedrigkeit”, „Kohäsion der Formativgruppe", d.h. ihre Festigkeit (die unter anderem durch morphologische und syntaktische Besonderheiten deutlich wird wie Flexionslosigkeit, Transformationsbeschränkungen usw.), und „Figuriertheit”, d.h. eine nicht aus der Komposition der einzelnen lexikalischen Elemente erklärbare Bedeutung (vgl. z.B. Gréciano 1983, S. 233-235). Bei der Anwendung dieser Kriterien erfährt man zwangsläufig erneut die Abgrenzungs- und Klassifikationsprobleme der strukturellen Phraseologie als Auffindungs- und Interpretationsprobleme. Spruchformen, d.h. die sogenannten satzwertigen Formeln wie Sprichwörter, Maximen und Sentenzen sind in der Regel aufgrund ihrer Konstruktionsweise im Material als formelhaft erkennbar, insbesondere aufgrund ihrer NichtAnpassung an den lokalen Kontext. Eine ÄuBerung wie wo kän kläger is is $k a ̈ n$ rischter fällt aus diesem Grund auch einem Beobachter auf, der die Formel nicht kennt.

Etwas komplizierter liegt der Fall meist bei satzwertigen Formeln mit Kontextanbindung ${ }^{3}$ durch deiktische Ausdrücke und Pronomina wie so oder das, z.B. so ist das Leben oder das kommt davon. Auch hier lassen sich aufgrund einer feineren Analyse noch Eigenschaften der Nicht-Angepaßtheit an den lokalen Kontext in Form von semantischen Sprüngen nachweisen (z.B. Sprünge von konkreten Darstellungen zu vagen und übergeneralisierten Aussagen).

Die Erkennungsschwierigkeit ist noch größer in Fällen, in denen keine Anomalien des kontextuellen Bezuges auftreten wie z.B der krigg=de eisschrongg abgschlosse. Eine Entscheidung darüber, ob es sich um einen individuell formu-

3 Vgl. die Unterscheidungen in Burger et al. (1982, S. 39): „'Feste Phrasen' sind ganze phraseologische Sätze, wobei der textlinguistische AnschluB an den Kontext durch Pronomina oder sonstige verweisende Elemente gewährleistet ist. Sprichwörter und Gemeinplätze sind satzwertige Phraseologismen, die im allgemeinen nicht durch textlinguistisch-verweisende Elemente an die Textumgebung angeschlossen sind; unter funktionaler Perspektive handelt es sich um allgemeine Aussagen oder Urteile, mit denen eine gegebene Situation erklärt, eingeordnet, beurteilt wird." 
lierten beschreibenden Satz oder um eine metaphorisch zu verstehende feststehende Formel handelt, ist nicht durch die Analyse dieser Äußerung alleine möglich. Die Formulierung enthält als Kernstück eine Lexemkombination den eisschrank abgeschlossen kriegen. Wie fest diese Kombination lexikalisch ist, d.h. von welcher Art die "Kohäsion der Formativgruppe” ist, ist nicht ohne weiteres zu sagen. Nicht belegt im Material ist z.B. die Aktiv-Umformung in jmdm. den eisschrank abschließen, belegt ist aber die Variante nicht an den eisschrank dürfen. Hier liegt eine feste Inhaltsfigur vor ('Eisschrank + Unzugänglichkeit'), die eingeschränkt variabel formuliert werden kann.

Derartige Probleme häufen sich natürlich im Bereich der phraseologischen Peripherie, d.h., wenn es um Derivate von bestehenden Formeln oder Analogiebildungen nach bestimmten Konstruktionsmustern oder um sprachlich noch nicht eindeutig verfestigte Stereotype geht, die als Formelreminiszenzen bzw. als Formelkandidaten verwendet werden. Die Phraseologieforschung weist mit aller Klarheit darauf hin, daß es unauflösliche Probleme der Abgrenzung und Klassifikation der Phraseologismen aus der systembezogenen, im engeren Sinne lexikalischen Perspektive gibt und daB neben dem Kernbereich der klaren Zugehörigkeit zur Phraseologie ein großer Bereich von kaum endgültig entscheidbaren Fällen besteht. ${ }^{4}$

Das Kriterium der "Gebräuchlichkeit” bzw. „Reproduziertheit”, das z.B. Burger et al. in die Definition aufnehmen ${ }^{5}$, ist bei der Materialanalyse in vielen Fällen sehr wichtig. Das gilt insbesondere in den Fällen, in denen die Reproduziertheit von den Sprechern auch erkennbar markiert wird, z.B. durch Einleitungsformeln wie wie sagt mer als $(a l s=i m m e r)$ in wie sagt ma als * die milsch frißd mid aus de schissel. Als eine Form der wiederholten Rede hat das formelhafte Sprechen Gemeinsamkeiten mit der Redewiedergabe. Aber von dieser unterscheidet es, dab nicht auf singuläre Sprechereignisse Bezug genommen wird, sondern auf einen wiederholten, gewohnheitsmäBigen Gebrauch. ${ }^{6}$

4 Zu den Abgrenzungsproblemen vgl. u.a. Gréciano (1983, S. 238): „Allgemein wurden die Schwierigkeiten bei der Festlegung von Idiomatizität als Grenze der Klassenbildung auf semantischer Basis anerkannt. Die Forschung spricht von der NichtAdāquatheit rein syntaktisch-semantischer Konzepten .

5 Vgl. Burger et al. (1982, S. 1): „Phraseologisch ist eine Verbindung von zwei oder mehr Wörtern dann, wenn (1) die Wörter eine durch die syntaktischen und semantischen Regularitäten der Verknüpfung nicht voll erklärbare Einheit bilden, und wenn (2) die Wortverbindung in der Sprachgemeinschaft, ähnlich wie ein Lexem, gebräuchlich ist. Die beiden Kriterien stehen in einem einseitigen Bedingungsverhältnis: wenn (1) zutrift, dann auch (2), aber nicht umgekehrt ${ }^{n}$.

6 Bei der Formelverwendung bilden zitathafte und damit auffallig gemachte und routinisierte, unauffällige Verwendung die Pole einer Skala. Diese Art der Auffälligkeit ist nicht identisch mit der Auffälligkeit im Sinne von "Abweichung von der vorgegebenen Formel ${ }^{n}$ in der psycholinguistisch orientierten Forschung; vgl. hierzu die Gleichsetzung von Burger et al. (1982, S. 189) „unauffalliger Gebrauch” = „richtiger Gebrauch". 
Darüber hinaus erweisen sich bei der Analyse des formelhaften Sprechens im natürlichen Vorkommenszusammenhang prosodische Eigenschaften und interaktiv textuelle Muster als zentral. Diese werden in der strukturell lexikologisch orientierten Phraseologie nicht berücksichtigt. Gerade für die Erfassung der phraseologischen Peripherie und der soziospezifischen Formeln mit relativ engem Verbreitungsgebiet liegt hier häufig aber gerade der Schlüssel. So indizieren vielfach prosodische Merkmale einen Kontrast der formelhaften Äußerung zum Kontext und markieren durch Auffälligkeiten der Sprechweise auch die Reproduziertheit. Wir werden diesen Phänomenen in unserer Analyse besondere Aufmerksamkeit widmen. ${ }^{7}$

(b) Phraseologische Merkmale und Formulierungsverfahren

Nicht nur die mehr oder weniger mustergetreue Verwendung geprägter Formeln ${ }^{8}$ sondern auch der fortgesetzte Prozeß der Formelprägung ist fester Bestandteil der alltäglichen Sprachpraxis. Für eine Untersuchung der Muster und Verfahren der Formelprägung gibt es eine Reihe von Anknüpfungspunkten in der vorliegenden Forschung. Einmal ist es möglich, die für die Typologie von Phraseologismen benutzten Mustervorstellungen auch als Regeln oder Anleitungen für Bildungsmuster zu formulieren. Für einige der Klassen in der strukturell-semantischen Mischtypologie von Burger et al. (1982) bietet sich das an, so ohne Zweifel für die sogenannten Paarformeln. Für Sprichwörter und Gemeinplätze sind solche Bildungsregeln auch dargestellt worden (vgl.z.B. Peukes 1977; Gülich 1978/1981). Wichtig in diesem Zusammenhang ist auch das Konzept der 'Phraseoschablonen' von Fleischer (1982). Phraseoschablonen sind syntaktische Strukturen - sowohl nicht prädikative Wortverbindungen als auch Satzstrukturen -, deren lexikalische Füllung variabel ist, die eine Art syntaktischer Idiomatizität aufweisen. Das syntaktische Konstruktionsmodell hat eine vom entsprechenden nicht-idiomatischen Modell abweichende, irreguläre Bedeutung (S. 196). Solche Schablonen sind zum Beispiel:

- $x$ ist $x$ (z.B. Urlaub ist Urlaub);

- Wiederholungen des Verbs mit und, z.B. will und will nicht enden;

- Personal-/Demonstrativpronomen + und + Substantiv mit unbestimmtem Artikel, z.B. du und ein versierter Autofahrer.

7 Gerade wenn man anhand von Gesprāchsmaterialien formelhaftes Sprechen zu identifizieren versucht, wird einem die Bedeutung der Sprechweise sofort deutlich. Es fällt auf, daß praktisch in der gesamten Phraseologie-Forschung die Sprechweise ausgeklammert worden ist; vgl. aber immerhin den Hinweis von Hain (1951) auf rhy thmische Muster.

8 Auf die Variation als Folge der eingeschränkten Formeltreue der Sprecher wird in der Literatur immer wieder hingewiesen: vgl. z.B. Quasthoff (1983) oder Gréciano (1983, S. 237): „Die jüngsten Auseinandersetzungen mit Phrasenverwendungen zeigen, wie sehr diese fixierten Einheiten im System den Sprecher zu kreativen Überschreitungen herausfordern. Bedeutungsfluktuationen gehören zum reizvollen Charakteristikum phraseologischer Vorkommnisse in Rede und Text". 
Diese Schablonen gestatten, immer neue Formulierungen mit formelhaftem Charakter zu bilden. Fleischer stellt den Modellen der Wortbildung ${ }^{9}$ phraseologische Modelle wie etwas staunen (z.B. Bauklötze staunen), jemandem auf etwas gehen, jemanden um etwas wickeln an die Seite (1982, S. 198ff.). ${ }^{10} \mathrm{Zu}$ dieser Annahme der Modelliertheit von Phraseologismen ist eine Gegenposition formuliert worden, welche die Variabilität und Produktivität der Phraseologie auf Eigenschaften des Gebrauchs zurückführt:

„Die progressive Verlagerung der Diskussion auf allgemein linguistische Grundfragen mit der Konzentration auf das immer schon latente semantische Problem stützt die These der Unmodelliertheit von Phraseologismen. Die vor allem inhaltliche, aber auch formale Varianz von Phraseologismen muß als einzige Invariante anerkannt werden. ... Erst die Auseinandersetzung mit den Formen des Sprachgebrauchs konnte diesen Aspekt der Phraseologie ins BewuBtsein rücken" (Gréciano 1983, S. 241). ${ }^{11}$

Zur Stützung dieser Position wird es darauf ankommen, Prinzipien und Verfahren der Sprachverwendung anzugeben, welche die Bildbarkeit und Verstehbarkeit von Phraseologismen erklären können. Zu diesem allgemeinen Problem kann unsere Untersuchung einige Beobachtungen beitragen.

Wir sind bei unseren Analysen unter anderem daran interessiert, welche Rolle formelhaftes Sprechen im Kontext des fortgesetzten Redens über die Welt in einer Gruppe spielt und wie sich im Verlauf des fortgesetzten Thematisierungsprozesses formelhafte Ausdrucksweisen herausbilden. Das ist mit Sicherheit ein gutes Beobachtungsfeld für die Untersuchung von Regeln bzw. Formulierungsverfahren für formelhaftes Sprechen. Bei diesen Prozessen der Formelbildung und -abwandlung spielen Muster eine große Rolle, die direkt oder analog reproduziert werden. Zusätzlich gibt es aber unserer Meinung nach Formulierungsverfahren von relativ allgemeiner Natur, die erst in Kombination Formelhaftigkeit hervorbringen und die in unterschiedlichem Grade angewendet werden können, so daB sie gleitende Übergänge von freier Formulierung zu formelhaftem Sprechen gestatten. Unsere Vorstellung dabei ist, daB mithilfe von Formulierungsverfahren gerade die Variabilität des mehr oder minder formelhaften Sprechens erklärt werden kann.

9 „Ein Wortbildungsmodell ist ein morphologisch-syntaktisch und lexikalisch-semantisch bestimmtes Strukturschema, nach dem Reihen gleichstrukturierter Wortbildungskonstruktionen mit unterschiedlichem lexikalischem Material produziert werden können" (Fleischer 1982, S. 196).

10 Die semantische Modellierung gründet in allgemeinen "logischen und assoziativen Prozessen des menschlichen Denkens" (Fleischer 1982, S. 201).

11 „Die Vorhersagbarkeit und Verallgemeinerungsfähigkeit von Phraseologismen werden dadurch beeinträchtigt, daß die im phraseologischen Sprachzeichen teilweise eingebüßte Autonomie der Formative im phraseologischen Sprachgebrauch unter bestimmten Bedingungen und mit gewissen Funktionen wieder hergestellt werden kann. Es handelt sich jedoch dabei nie um referentielle Transparenz des wörtlichen, sondern um seine pragmatische Umfunktionalisierung" (Gréciano 1983, S. 241). 
(c) Formelhaftes Sprechen und Wissensorganisation

Bei unserem Vorgehen spielt der Zusammenhang des formelhaften Sprechens mit Thematisierungsprozessen in Sprechergemeinschaften und den dadurch manifestierten und immer wieder präsent gehaltenen Wissensbeständen eine wichtige Rolle. Auf diesem im engeren Sinne sozio- oder ethnolinguistischen Gebiet besteht ein deutliches Defizit. Derartige Zusammenhänge sind am ehesten in der Stereotypen-Forschung beachtet worden. Es gibt mit Sicherheit „eine Übergangszone zwischen dem sozialwissenschaftlich definierten Stereotyp und dem linguistischen Bereich der Phraseologie: die Sprichwörter und sprichwörtlichen Gemeinplätze, sofern sie in den Sprachbesitz als vorgefertigte Formulierungen eingegangen sind" (Burger et al. 1982, S. 8), aber das gilt nicht nur für Sprichwörter und sprichwörtliche Gemeinplätze. Ein wichtiger Anknüpfungspunkt ist sicher auch der Ansatz von Bernstein mit dem Versuch, einerseits unterschiedliche Grade und Arten von Formelhaftigkeit und Idiomatizität sozialstilistisch einzuordnen (und zwar nach sozialen Schichten) und andererseits Formelhaftigkeit in Zusammenhang zu bringen mit Fragen der Ausdrucks- und Argumentationsfähigkeit. ${ }^{12}$ Wir knüpfen an die Vorstellung der sozialstilistischen Unterschiede an, ohne aber die Hypothese vom Zusammenhang von Stereotypie und kognitiver Kompetenz zu übernehmen. Um der Gefahr der einseitigen Verallgemeinerung von sozialstilistischen Merkmalen zu entgehen, ist es notwendig, unterschiedliche Kommunikationstypen und Interaktionsmodalitäten in Betracht zu ziehen und jeweils die Funktionalität des formelhaften Sprechens bezogen auf diese Kontexte zu untersuchen.

\section{(d) Funktionen und Verwendungsbedingungen von sprachlichen Formeln}

Inzwischen liegt eine Reihe von Untersuchungen zu den Funktionen des formelhaften Sprechens vor. Hervorgehoben werden dabei Funktionen der Gesprächsorganisation (von sogenannten Routineformeln bzw. pragmatischen Idiomen; vgl. u.a. Gülich/Henke 1979/1980; Coulmas 1981a), der Konsensherstellung durch die Formulierung von kollektiv akzeptierten Vorstellungen, insbesondere in der Form von Sprichwörtern, Gemeinplätzen und sprachlich nur bedingt verfestigten sozialen Stereotypen (vgl. Quasthoff 1973; Wenzel 1978; Gülich 1978/1981; Schröder 1988) und der Regulierung sozialer Distanz durch Höflichkeit (vgl. u.a. Gülich/Henke 1979/1980; Colmas 1981a). Daneben gibt es auch

12 Zur Soziolinguistik von Stereotyp und Phraseologie vgl. Bernstein (1973, S. 133): „Eine 'óffentliche Sprache' enthălt eine große Anzahl überlieferter idiomatischer Wendungen, aus denen der einzelne auswählt. Anstelle des individuellen Lernens, das einen Sprachgebrauch schafft, innerhalb dessen man auswählen kann, um die eigenen individuellen Gefühle auszudrücken, mißt der der 'öffentlichen Sprache' Verhaftete seine Gefühle sozialen Stereotypen bei, die die Solidaritāt sozialer Beziehungen auf Kosten der logischen Kommunikationsstruktur und der Besonderheit des Fühlens vergrößern”. Diese frūhen Uberlegungen Bernsteins sind in der Soziolinguistik bislang nicht systematisch weiter verfolgt worden (vgl. auch Burger et al. 1982, S. 8/9). 
Hinweise auf einen Zusammenhang zwischen Sprechhandlungstypen und bestimmten Phraseologismen; so weisen Burger et al. (1982) auf das sogenannte "symbolische Versprechen” (z.B. ich freß einen Besen) hin sowie auf den phraseologischen Charakter vieler Versprechen, Befehle, Warnungen und Drohungen (z.B. reiß dich am Riemen; wetten, daß usw.; Burger et al. 1982, S. 110ff.). Wir werden derartige Überlegungen insbesondere für formelhaftes Sprechen im Rahmen von Tratsch, d.h. des Redens über Dritte verfolgen. ${ }^{13}$

Erst sehr wenige Hinweise gibt es auf den Zusammenhang von formelhaftem Sprechen und Redekonstellation bzw. Regeln des Sprechens (wann ist welche Art von formelhaftem Sprechen angemessen?). ${ }^{14}$ In unserer Analyse ergeben sich in dieser Hinsicht insbesondere Beobachtungen zum Zusammenhang von formelhaftem Sprechen und sozialer Konstellation in der beobachteten Gruppe, insbesondere im Hinblick auf die Unterscheidung $z w i s c h e n$ der Kerngruppe und später Hinzugekommenen sowie den externen Teilnehmern (der Ethnographin und der offiziellen Betreuerin).

Eine besondere Rolle spielt für unsere Analyse die Funktion des formelhaften Sprechens als Kontextualisierungshinweis (vgl. Gumperz 1982a). Mit der erkennbar gemachten Formelhaftigkeit seiner Äußerung weist ein Sprecher auf vorausgehende Verwendungen bzw. einen Verwendungszusammenhang hin. Formelhaftes Sprechen ist insofern geeignet, Diskurswelten zu indizieren, aus denen die Formeln stammen, in denen sie gebräuchlich sind und eine besondere Funktion haben. Die feste Geprägtheit von beim fresse lernd ma die leid kenne z.B. verweist auf typische Verwendungskontexte, in denen "einfache Leute" zwischen echten und falschen Vornehmen unterscheiden und diese entlarven (vgl. auch Beitrag 3, Kap. 5.2.). Der Verwendungszusammenhang kann auch durch generalisierte Formeln der Redeeinleitung ausgedrückt werden: „wie wir immer sagen”, „wie alle vernünftigen Menschen sagen”, „wie die feinen Leute sagen”, „wie man in Mannheim sagt" usw. Die verweisende Funktion des formelhaften Sprechens ist unabhängig davon, ob die soziale Bezugnahme in redeeinleitenden Formeln explizit gemacht wird oder nicht. Aufgrund dieser Bezugnahme kann Formelhaftigkeit als ein Verfahren der Kontextualisierung angesehen werden, d.h. als ein Hinweis darauf, welche spezifischen Wissensbestände für die Interpretation einer Äußerung herangezogen werden sollten. ${ }^{15}$

13 Vgl. auch Burger et al. (1982, S. 120ff.) zur Analyse der Phraseologismen als eine dominante Formulierungsweise beim Kartenspiel. Die Betrachtungsweise von Phraseologismen im Rahmen von bestimmten Handlungskomplexen entspricht der Ausrichtung unserer Analyse von Charakterisierungssequenzen.

14 Zum Verhältnis von Phraseologie und Redekonstellation vgl. auch die Beobachtungen von Hain (1951) zur „normalen” Verwendung von Sprichwörtern durch die Alten und die markierte, erklärungsbedūrftige Verwendung durch Jüngere.

15 Ansātze zu einer Betrachtung der kontextualisierenden Funktion in der Phraseologie kann man auch aus Bemerkungen darüber herauslesen, daß das Erkennen des Quellenbezuges von Phraseologismen, insbesondere von geflügelten Worten, für de- 


\section{Formulierungsverfahren für Charakterisierungsformeln}

Das Ziel ist hier, Formulierungsverfahren anzugeben für formelhafte Charakterisierungen von Individuen, soziale Beziehungen und Situationen, wobei jeweils der konkrete Fall oder die soziale Kategorie im Vordergrund stehen kann. ${ }^{16}$ Typische Fälle von derartigen konkreten Charakterisierungen von Individuen sind:

- der krigg=de eisschrongg abgschlosse

- er kriggd nudle mid schinke sie frißd zwee schdeeks

- er gehd hääm mach=die bedde * sie gehd in die lokale zum schnorre.

Es handelt sich um Formulierungsverfahren, die im Prinzip in zwei Richtungen angewendet werden können bzw. zu denen jeweils komplementäre Verfahren gehören, die eine Ausprägung oder Veränderung der Formulierung in die entgegengesetzte Richtung bewirken. Die Anwendung in eine Richtung steigert die Formelhaftigkeit, die Anwendung in die andere Richtung löst den Formelcharakter auf und steigert den Charakter von variablen, in der Situation produzierten Formulierungen.

Der Einfachheit halber stellen wir in der folgenden Übersicht die Verfahren nur in einer Richtung dar, und zwar als Verfahren zur Herstellung von Formelhaftigkeit. Die Anwendung dieser Verfahren ist sowohl innerhalb eines Gesprächs beim Reden über einen Gegenstand zu beobachten als auch in der Kommunikationsgeschichte der Gruppe.

\subsection{Relevanzsetzung und Zuspitzung}

Die hier zu behandelnden Charakterisierungen sind prägnant. Dieses Gestaltungsmerkmal wird durch die Kombination unterschiedlicher Verfahren erreicht: Auswahl von wesentlichen Eigenschaften und Steigerung der relevanten Züge durch Zuspitzung. Die Auswahl der Eigenschaften wird einerseits durch 'Salienz', d.h. das Hervortreten auffäliger Eigenschaften gesteuert, andererseits durch ihren Bezug auf wesentliche soziale Kategorien. Gutes Material für die Formelbildung sind auffälige und einprägsame Eigenschaften und Verhaltensweisen, die als kategoriengebunden anzusehen sind (d.h. als definierend für bestimmte soziale Katagorien; vgl. insbesondere Beitrag 5). Das Ausgangsmate-

ren Interpretation von Bedeutung ist (vgl. Burger et al. 1982, S. 43ff., S. 47). Weiter kōnnen auch Beobachtungen zur stilistischen Qualitāt von Phraseologismen (vgl. Fleischer 1982) oder auch zu Floskeln und Redewendungen als gruppensprachliche Merkmale einbezogen werden.

16 Die Darstellung der einzelnen Formulierungsverfahren stützt sich z.T. auf die Untersuchung zum Erzāhlen in Kallmeyer/Schütze (1977); vgl. auch Schütze (1987a), Kallmeyer (1981). Es handelt sich um allgemeine Verfahren wie Detaillierung, Kondensierung, Konturierung, GestaltschlieBung, die beim Erzāhlen einen besonderen Status gewinnen und als Zugzwānge fortlaufend wirken, solange die Darstellung der kognitiven Figur der Geschichte und ihrer Komponenten läuft. 
rial für Charakterisierungsformeln findet sich vielfach in Erzählungen, in denen die relevanten Eigenschaften von sozialen Einheiten breiter und mit komplexeren Typisierungen herausgearbeitet werden. Ein Mittel der Steigerung ist die Zuspitzung der Darstellung durch die Auswahl sprechender Details und Übertreibungen. Eine solche Zuspitzung auf ein sprechendes Detail enthält z.B. halwi dafel schoklad zur Darstellung einer knauserigen Zuteilung von GenuBmitteln in un wenn=a brav is kriggd=a halwi dafel schoklad wehe wenn=a mehr will. In diesem Sinne ist auch durch die Wahl von abschließen die Formulierung der krigg=de eisschrongg abgschlosse prägnanter als de aame Oddo ho=do gar ned on de kischeschrongg gedirfd. Der Konturierung dienen Oppositionsbildungen, z.B. zwischen "er" vs. „sie":

- er gehd hääm mach=die bedde sie gehd in die lokale zum schnorre

- der schbield=s gscherr macht alles sie hod ihr fingernäggl dud sisch pflege.

Solche Oppositionsbildungen sind ein häufig verwendetes Konstruktionsmuster, auch zur Darstellung komplexerer Charakterisierungen. So charakterisiert ein Gruppenmitglied eine ältere Frau durch:

die brauchd vielleischd a schdunn

fer in die schdrobebohn noi

avier venn=s donze $\uparrow$

do brauchd se kään schdegge $\uparrow$

do brauchd se nix $\downarrow$

\subsection{Kondensierung und GestaltschlieBung}

Formelhafte Charakterisierungen sind kurz. Sie haben entweder das Format von einfachen Sätzen, die z.T. noch elliptisch verkürzt sind, oder von zweiteiligen, maximal dreiteiligen Satzgefügen mit kurzen Sätzen und einem klaren, geschlossenen Verknüpfungsschema (wenn - dann, er vs. sie o. ä.).

Die verwendeten Formelversionen sind unterschiedlich kondensiert bzw. expandiert. Reduzierte Formulierungen erscheinen vielfach in Sequenzen des formelhaften Sprechens in den Reformulierungen erster Charakterisierungen:

der $k r i g g=d e$ eisschrongg abgschlosse daB=a ned zuviel friBd

der kriggd de eisschrongg abgschlosse

der kriggd=n abgschlosse.

In diesem Fall wird mit den Reformulierungen die Kondensierung verstärkt. Neben dieser fortschreitenden Anwendung der Kondensierungsverfahren gibt es auch im Rahmen solcher Sequenzen formelhaften Sprechens Expansionen, die der maximalen Kondensierung entgegenlaufen: $u n$ wenn=a brav is kriggd=a halwi dafel schoklad $\downarrow$ we"he wenn=a mehr will. Das letzte Formulierungssegment we"he wenn=a mehr will stellt eine Expansion im Verhältnis zu einer möglichen minimalen Formulierung un wenn=a brav is kriggd=a $\ddot{a}$ halwi dafel 
schoklad dar. Diese Expansion expliziert bereits in der minimalen Formulierung (speziell in wenn er brav is) implizierte Bewertungen und Sachverhaltsmerkmale.

Die Charakterisierungsformeln sind als geschlossene Einheiten formuliert; sie sind in sich vollständig. Diese Geschlossenheit der Äußerungseinheit wird durch verschiedene Mittel deutlich gemacht. Syntaktisch und intonatorisch sind alle Äußerungen als abgeschlossen markiert; es gibt keine auslaufenden ÄuBerungen, d.h. syntaktisch unvollständige ÄuBerungen, bei denen der Sprecher durch Dehnungen und sinkende Intensität anzeigt, daß er bereit ist, das Wort abzugeben. Die Äußerungen werden außerdem relativ schnell und in einem Zug formuliert, d.h. ohne Verzögerungen und ohne starke Zäsuren. Bei mehrgliedrigen Äußerungen wird die Geschlossenheit ebenfalls durch die Sprechweise markiert; sie enthalten nur wenige und nur relativ schwache Zäsuren im Sprechfluß. Tendenziell werden sie in einem Zug formuliert:

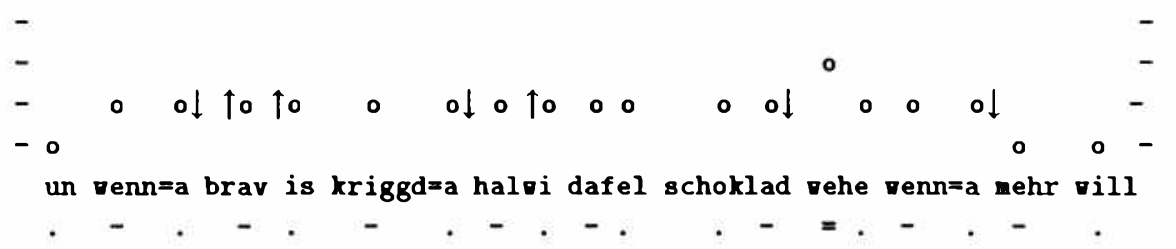

Bei mehrgliedrigen Konstruktionen wie den oppositiven Darstellungen, die eine Zäsur zwischen den beiden Segmenten erfordern, wird die Zusammengehörigkeit zu einem Format durch prosodische Merkmale wie parallelen Rhythmus der Formulierungssegmente und korrespondierende Intonationsbögen (aufsteigender und absteigender Ast) deutlich gemacht.

Es gibt noch einen weitergehenden Aspekt der Kondensierung: Die formelhaften Charakterisierungen (ebenso wie die speziellen Maximen) gehen in der Regel auf Geschichten zurück. Die kurzen Charakterisierungen kommen durch die Auswahl von Kernelementen von Erzählungen, Szenenhöhepunkten usw. und deren fortschreitender Verkürzung zustande. Eine wichtige Zwischenstufe zwischen Geschichten und Charakterisierungsformeln sind Geschichtenkondensate. Sie sind ein sehr häufiges Format im Tratsch. Solche Kondensate sind z.B. die folgenden generalisierten Szenendarstellungen. Zur Charakterisierung von Gerdas Unverfrorenheit äuBert Frau Müller:

die sacht zu demm Oddo|

geh ä schdund nunner in de keller $\uparrow$ *

un in=ere schdunn konnsch vidder ruffkumme-

in der zeit hot se=n onnere

(2036/62)

Frau Zimmermann kennzeichnet im selben Kontext die Verblendung von Otto durch eine in erlebte Rede transformierte Auseinandersetzung mit ihm: 
der is verhei"rad $\uparrow$ mid=ere

un der därf/

<bei denn därfe se nix sa:che $\uparrow$ nei:n†

des is seine frau" $\downarrow$

Und Frau Müller bei anderer Gelegenheit:

die hod als onnere do [...]

sag=der Oddo nix [...]

guggd in die noi vie in schbiggel

$(2036 / 62 a)$

Diese generalisierten Szenendarstellungen erscheinen mit Redewiedergabe und auch als Formulierung in der dritten Person. Tendenziell haben die Formulierungen in der dritten Person eine stärkere Affinität zur Kondensierung: Redewiedergabe ist in Erzählungen ein Mittel der szenischen Darstellung und damit der Detaillierung; die Umformung zu einem narrativen oder deskriptiven Satz ist dagegen ein Mittel der Kondensierung. Geschichtenkondensate haben meistens eine dreigliedrige Darstellungsstruktur, in der Regel mit einer Pointe oder einer Bewertung bzw. einem Kommentar in der dritten Position.

\subsection{Kommentarformat}

Die eindeutig formelhaften Charakterisierungen haben die Struktur von Kommentaren, d.h. von Äußerungen, welche die Thematisierung eines Bezugssachverhalts voraussetzen und zu diesem beschreibend und urteilend Stellung nehmen. ${ }^{17}$ Ausschlaggebend für die formelhaften Kommentare im Tratsch sind folgende Merkmale:

- die Kennzeichnung eines vorerwähnten thematischen Referenten, in der Regel durch ein akzentuiertes Demonstrativpronomen (z.B. de" $r$ ) in der ersten Satzposition;

- das Format eines Aussagesatzes;

- Formulierung in der dritten Person - keine explizite Sprecherreferenz (z.B. in Rahmensätzen wie ich find, ich meine usw.).

Kommentare dieser Art, die in unterschiedlicher Weise die übrigen Eigenschaften des formelhaften Sprechens aufweisen, sind z.B.:

- der hod alles schaffe misse ohje ohje

- der arbeided nix

- der wär nimmer kumme.

17 Unsere Definition von Kommentaren lehnt sich an Posner (1972, S. 25) an: „Gegeben sei eine Folge zweier Informationen, Fj und Fk. Tritt eine Teilinformation $\mathrm{Fi}$ von $\mathrm{Fj}$ in $\mathrm{Fk}$ als [prädikatenlogisches] Argument auf, so nennen wir Fk 'einen Kommentar von Fj' und Fi 'das Kommentat'. Fj bezeichnen wir als 'Kommentandum' und die Folge (Fj,Fk) als 'Kommentierung'. Der Vorgang der Hinzufügung eines Kommentars zu einem Kommentandum heibt 'Stellungnahme'" 
Die formelhaften Charakterisierungen sind Folgeäußerungen, die auf eine Vorgängeräußerung reagieren. Die Vorgängeräußerung kann den AnlaB zur Reaktion gezielt schaffen und damit die Fortsetzung mit einer formelhaften Charakterisierung provozieren; sie kann in einem schwächeren Sinne aber auch nur die Gelegenheit zu einer solchen Reaktion bieten. ${ }^{18}$ Die Kommentare reagieren unmittelbar auf die BezugsäuBerung, d.h., sie erscheinen normalerweise in der nächstfolgenden Äußerung. Die Bezugsäußerung führt den thematischen Referenten ein - dann ist der Kommentar die erste Themenfortführung - oder sie gehört zu einem Komplex von Äußerungen, die den Referenten thematisieren. Es gibt aber auch die - viel seltener benutzte - Möglichkeit, beide Schritte in eine Äußerung zu integrieren. Dabei folgen eine 'links herausgestellte Thematisierung' ${ }^{19}$ und eine auch prosodisch deutlich davon abgesetzte Charakterisierung aufeinander: un der ihr mann $\uparrow$ des=s au"ch so=n daggll. In diesem Fall hat die Charakterisierung noch ein Thematisierungsmerkmal (im vorliegenden Fall auch; vgl. weiter unten).

Schließlich werden die Kommentare als Reaktionen auf vergleichbare Kommentare verwendet; das ergibt dann Sequenzen kollektiven formelhaften Sprechens. Einen solchen Fall zeigt das folgende Beispiel. Die erste Äußerung führt das Thema "Otto" ein, nachdem vorher über Gerda geredet worden ist:

S. 2

20 HI: un der ihrn mann $\uparrow$ des=s auch

K : LACHEN

S. 3

$12 \mathrm{I}$ :

des is: $n$ amer

$2 \mathrm{SU}$ :

$\rightarrow a^{\prime \prime c h ~ d i e ~ h a t ~ a ~ n o c h=e n ~ m a n n ~} \uparrow$

3 мӥ:

de:r hod nix zu melde

4 HI : so=n daggl $\downarrow$

$\mathrm{K}$ :

DURCHEIMANDER

5 ZI: deifel $\downarrow$

desm aamer deifel $\downarrow$

6 MÜ:

virklisch $\downarrow$

18 Die Abstufung der Vorstrukturierung von Folgeāußerungen durch die jeweilige Äußerung - z.B. von der Obligation als starker Bedingungssetzung über Anlaß bis Gelegenheit als schwacher Bedingungssetzung - ist bislang wenig behandelt. Der Begriff 'Paar' in der Konversationsanalyse bezeichnet eine relativ starke Beziehung: Die erste Āußerung macht als „first pair part” eine Folgeäußerung als „second pair part" erwartbar (vgl. Schegloff/Sacks 1973). Neben dieser Beziehung gibt es wesentlich offenere Beziehungen zwischen zwei Āußerungen in der angedeuteten Art.

19 In der Terminologie von Altmann (1981) handelt es sich dabei um ein 'freies Thema', das sich von anderen Formen der Herausstellung durch die Merkmale unterscheidet: NP im Nominativ kombinierbar mit Einleitungsausdrūcken, steigende Intonation, Abtrennung vom Folgesatz durch eine Pause. Vgl. auch die 'nominale Setzung' in Schrōder (1987). 
$7 \mathrm{HI}$ :

das der/

daß der $n i=$ der zusammeleve

$\mathrm{K}$ : LACHEN

(Text 2036/62)

Die thematische Einführung von Otto $(2,20-3,4)$ löst eine schnelle Sequenz von stereotypen und zweifelsfrei geteilten Bewertungen aus, die - bis auf eine Ausnahme, und zwar die Äußerung von Frau Schumann (SU) in 3,2-deutlich parallel formuliert werden: inhaltlich stimmen sie überein, syntaktisch übernehmen sie den Konstruktionsrahmen der ersten Charakterisierung (des $=s$ auch so $=n$ daggl), die Intonation fält in paralleler Weise, und es gibt starke rhythmische Übereinstimmungen durch die Akzentverlagerung nach links (der stärkste Akzent liegt in keinem Fall auf dem Typisierungsausdruck am Äußerungsende:

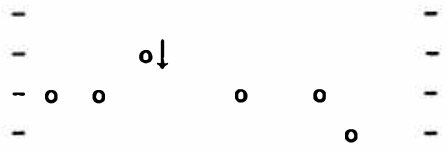

HI : $\operatorname{des}=8$ au"ch so=n daggl

- $\quad=$

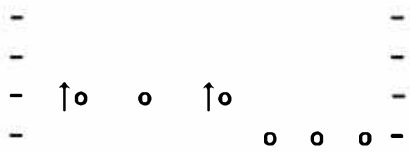

MÜ: de:r hod nix zu melde

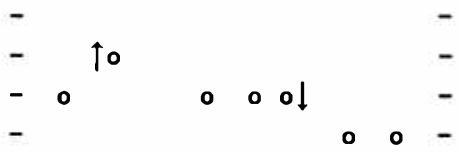

2I: des is: $n$ aamer deifel

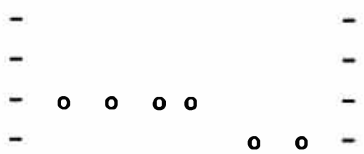

ZI: des=n aamer deifel 


\subsection{Entindexikalisierung}

Entindexikalisierung ist die Tilgung von expliziten Verweisen auf konkrete raum-zeitlich verankerte Situationen. Die personale Deixis bleibt bei den Personencharakterisierungen natürlich erhalten. Es erscheinen aber keine expliziten Verweise auf konkrete historische Situationen wie „damals”, „als Gerdas Mutter noch lebte" o.ä., wie sie gerade für Erzählungen charakteristisch sind. Und mit Ausnahme von den Fällen, in denen über eine historische Welt gesprochen wird, ist die Entindexikalisierung mit der Transposition in das Präsens verbunden. Im Kontrast zu den formelhaften AuBerungen wie die schreid=n $\tilde{o}$ der derf nix redde zeigt auch ein Erzählbeginn wie mir ware mol fordd bei de/ do war die fra Kattowitz dabei noch die indexikale Verankerung als konstitutives Merkmal von Erzählungen, wenn auch bezogen auf einen Situationstyp „Ausgehen”, der nur durch mol (=einmal) und den Hinweis auf die historisch spezifische personale Konstellation (die Anwesenheit der fra Kattowitz) individualisiert wird. In Ubereinstimmung mit dieser Herauslösung aus historisch verorteten Ereigniszusammenhängen fehlen auch Lokalisierungen in einer Ereignisfolge ( und dann, und da), wie sie ebenfalls für das Erzählen konstitutiv sind. An die Stelle dieser Einbettung treten u.a. wenn-dann-Konstruktionen.

Ein weiteres Beispiel für die Herauslösung eines Szenenkerns aus einer Geschichte und seine ansatzweise Verarbeitung zu einer generalisierten Formel für ein Handlungsprinzip zeigt das Reden über eine Frau aus der Filsbach, die sich eine Zeitlang Geld als Tagesmutter verdient hat und dabei die fremden Kinder im Unterschied zu ihrem eigenen (Gabi) grob vernachlässigt hat. Hier ist der ProzeB der Herstellung einer Formel an einer Folge von Reformulierungen innerhalb eines Thematisierungsbogens $\mathrm{zu}$ erkennen:

1 MÜ: bloß hod se=s in=s egg hinnere gsetzt hod erre e brezzel in die

2 mü: hond gevve e troggeni un die Gabi die hod die hot=s eis kriggd

$[\ldots]$

$21 \mathrm{KU}$ : die kinner have e brezzel in die hond gedriggd un die onner

$22 \mathrm{KU}$ : hod e portion eis kriggd

$[\ldots]$

$31 \mathrm{KU}$ : do toinemme un donn kriggt se e schdigg brezzel in die hond $\mathrm{K}$ : $\quad$ =GELD EINHEHMEN*

$32 \mathrm{KU}:$ gedriggd

Auf der ersten Stufe sind noch alle wesentlichen Merkmale der indexikalen Einbettung vorhanden, auf der zweiten Stufe in der Reformulierung durch Frau Kunz hat schon eine deutliche Kondensierung stattgefunden; die Situationsumstände (in=s egg gsetzt) sind getilgt ebenso wie Spezifizierungen (e troggeni); 
auf der dritten Stufe wird mit der Umformung ins Präsens eine Generalisierung der Handlung zur Handlungsweise durchgeführt.

\subsection{Generalisierung}

Bei den Charakterisierungsformeln handelt es sich um Aussagen über Individuen und nicht über soziale Gruppen oder Kategorien wie bei den sozialen Stereotypen; diese Aussagen können deswegen auch nicht die Form von Allsätzen haben, sie sind aber allgemeiner Natur: sie schreiben Eigenschaften und Handlungsweisen als typisch und immer geltend zu. Das ist auch für eine vergangene Welt als historische Generalisierung möglich, normalerweise im Material aber mit Formulierungen im Präsens verbunden. Die Generalisierung vollzieht sich in folgenden Stufen:

(a) Die Grundform ist ein singulärer indexikaler Satz weil er brav war, hat er eine halbe Tafel bekommen (Voraussetzung + Folge).

(b) Auf der nächsten Stufe wird dadurch generalisiert, daB Quantoren wie meistens oder immer eingesetzt werden und/oder daB die Voraussetzung im singulären Satz in den wenn-Teil einer wenn-dann-Konstruktion eingesetzt wird:

- Wenn er brav ist, bekommt er eine halbe Tafel Schokolade.

- Er bekommt meistens/immer eine halbe Tafel Schokolade.

Man kann hier von einer 'konditionalen Generalisierung' sprechen, bei der Situationstypen als Voraussetzungen in die Formel aufgenommen werden bzw. von einer 'empirischen Generalisierung', bei der durch die Verwendung von Quantoren noch ein Bezug zu Beobachtungen als Generalisierungsbasis hergestellt wird.

(c) Die dritte Stufe bildet die 'apodiktische Generalisierung', bei der die Voraussetzung und der Quantor getilgt sind:

- der kriggd=a halwi dafel schoklad

- sie gehd in werddschafle er muß hääm bedde mache.

Bei dieser letzten Form verdeutlicht die Voraussetzungslosigkeit und die fehlende Quantifizierung die bedingungslose Gültigkeit der zugeschriebenen Eigenschaft oder Handlungsweise.

\subsection{Metaphorisierung}

Im Zusammenhang mit einigen der voraufgehenden Operationen (insbesondere der Zuspitzung) bekommen die Formeln figurative Bedeutung. Der konkrete historische Hintergrund für der kriggd de eisschrongg abgschlosse ist, daB es in den 50er Jahren Eisschränke mit abschließbaren Türen gab, die aber dann aus Sicherheitsgründen nicht mehr hergestellt wurden; das "AbschlieBen” ist heute nicht mehr im konkreten Sinne zu interpretieren. Hier ist die Metaphorisierung besonders deutlich; aber auch die Präzision der Zeitangabe in un alle schdund 
eine zigaredd oder der Mengenangabe halwi dafel schoklad sind nicht im Sinne einer exakten Situationsbeschreibung relevant, sondern als Verdeutlichung der sparsamen und von Gerda strikt kontrollierten Zuteilung. Die Bedeutung der Äußerungen ist figurativ, aber noch sehr direkt auf konkrete Hintergründe bezogen. Ausgangspunkt der Formelbildung sind ganz konkrete Darstellungen, die formelhaften Charakterisierungen fußen auf unmittelbarer Wirklichkeitswahrnehmung. Die Nähe zur ursprünglichen Erfahrungssituation äußert sich in diesem Fall in der nur partiellen "Demotivierung:" ${ }^{20}$ Wie auch andere Formulierungen z.B. das spätere de aame Oddo ho=do gar ned on de kischeschrongg gedirfd zeigt, geht es wirklich um die Zugänglichkeit des Eisschranks; in der kriggd de eisschrongg abgschlosse ist metaphorisch in erster Linie das "AbschlieBen". Die Wirklichkeitsnähe gilt auch für die anderen Details von Ottos unfreiwilligem Konsumverzicht: die konkreten Objekte von Gerdas Zuteilung sind u.a. tatsächlich Zigaretten und Schokolade.

\subsection{Interaktionsmodalität der fraglosen Sicherheit}

Die eindeutig formelhaften Charakterisierungen haben hinsichtlich der von ihnen initiierten oder getragenen Interaktionsmodalität den Ausdruck der Selbstverständlichkeit, der fraglosen Sicherheit. Ein starkes Ausdrucksmittel dafür ist die Prosodie: wenig expressiv mit relativ weit links liegendem Intonationshöhepunkt und sinkender SchluBintonation. Besonders auffällig wird das bei Sequenzen kollektiven formelhaften Sprechens:

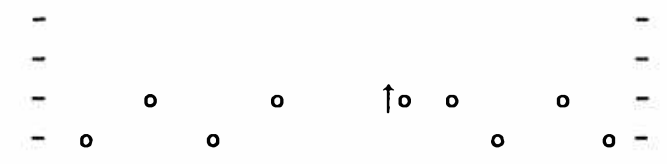

HE: der vär vielleisch gar nimmer kumme

$20 \mathrm{Zu}$ den Prozessen der Demotivierung und Remotivierung im Zusammenhang mit dem figürlichen, metaphorischen Charakter von Phraseologismen vgl. u.a. Gréciano (1983, S. 235): „U.E. läßt sich gemäß Sprachverwendung phraseologische Figuriertheit als obligatorische Demotivation, sowie zusätzliche fakultative Remotivation definieren, wobei sich beide Prozesse auf einen Teil (partielle De- oder Remotivation) oder auf alle Formative (totale De- oder Remotivation) erstrecken können. [...] Demotivation deckt sich mit der Verdunkelung des Wörtlichen, mit der Aufhebung der Referenz; Remotivation mit der Reaktualisierung von Transparenz, mit Literalisierungstendenzen. [...] Die Demotivationsphase der Figuriertheit entspricht dem 'Automatismus', wie er sich in allen Belegen des 'unauffalligen Gebrauchs' von PH äußert”. Remotivierung entspricht „auffäligem Gebrauch”." 


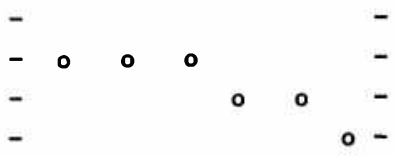

ZI : der vär nimmer kunme

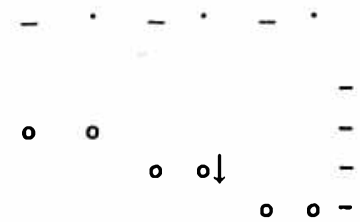

HE: der vär nimmer kumme

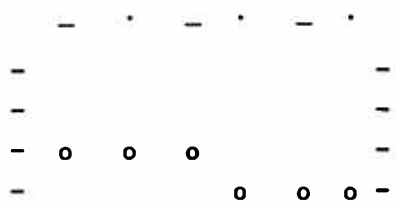

Mü: der vär nimmer kumme

Bei der internen Ausgestaltung von formelhaften Sequenzen erscheint auch eine Steigerung der Expressiviät (zusammen mit Expansionen), aber diese Expressivitätssteigerungen bleiben verhältnismäBig gedämpft: $u n$ wenn=a brav is kriggd=a halwi dafel schoklad we"he wenn=a mehr will. Die Hervorhebung von we"he existiert nur relativ zur insgesamt nicht-expressiven, planen Sprechweise und hat eher andeutenden Charakter.

In formelhaften Charakterisierungen fehlen:

- Ausdrücke des Zweifels oder der Möglichkeit (vielleicht), der Formulierungsunsicherheit (sozusagen), Faktizitätsbeteuerungen wie in des is tatsach daß die onnere hod (gemeint ist Gerdas Umgang mit Männern); bei fortschreitender Formelhaftigkeit einer Folge von Reformulierungen werden solche Ausdrücke regelmäBig getilgt: der wär vielleisch gar nimmer kumme wird zu der wär nimmer kumme;

- argumentative Konnektoren wie awwa, awwa trotzdem o.ä. wie in awwa trotzde:m demm wu=s so gemach werd demm gherd=s ned onnerschd;

- Betroffenheitsmarkierungen: also wi"rklisch;

- erregtes, emotional gefärbtes Sprechen.

Die auf diese Weise indizierte Interaktionsmodalität der fraglosen Sicherheit schlieBt Bemühungen um Differenzierungen der dargestellten Sachverhalte, Problematisierungen, Aktivitäten der Überprüfung von Urteilen und manifeste Anstrengungen der Bewältigung von emotionaler Betroffenheit aus. Damit 
nehmen die formelhaften Charakterisierungen eine spezifische Stellung im Spektrum der unterschiedlichen Interaktionsmodalitäten ein.

Beim Reden über die Welt kann sich das Interesse primär auf unterschiedliche Aspekte des Wissens über die Welt und des Redens über dieses Wissen richten: Es kann um Faktizität gehen, also darum, wie etwas ist/war bzw. ob es wirklich so ist/war; es kann die Arbeit des Typisierens, der Definition von Typen und der Bewertung im Vordergrund stehen; das Interesse an der Geteiltheit des Typisierungswissens in einer Gruppe kann als Motiv dominieren; und hin und wieder wird auch die eigene Kompetenz der sprachlichen Typisierung fokussiert und demonstrativ eingesetzt. Das sprachliche Verhalten, d.h. die Formulierungs- und Sprechweise sowie die Interaktionsformen sehen bei diesen Modalitäten jeweils unterschiedlich aus. Das Interesse an der Faktizität spielt z.B. eine besondere Rolle beim Erzählen historischer Ereignisse: des sin so erlebnisse wo ma äfach net vergißt. Dabei sind Zeugenschaft, Verbürgtheit und die Herstellung einer richtigen Version entscheidend. Rückt die Typenbildung in den Vordergrund, werden bestimmte Abstraktionen hinsichtlich der Vielfalt des Faktischen vorgenommen, und die Vorstellung von Typen gewinnt relative Eigenständigkeit; auf dieser Grundlage können dann konkrete Fakten, die nicht zum Typ passen, in der Darstellung ausgespart, verändert oder als erklärungsbedürftig behandelt werden.

Unsere Tratschmaterialien zeigen vielfältige Verschiebungen zwischen diesen Interaktionsmodalitäten. Die Ausprägung der Formeln, die wir hier analysieren, hat mit der Verlagerung der Modalität beim Reden über die Welt von der Faktizität zur Typizität zu tun. Darüber hinaus gibt es Formen des kollektiven formelhaften Sprechens, bei dem die Geteiltheit des Typisierungswissens in phatischem Sinn bestimmend wird, und teilweise zeigt die spielerische Ausgestaltung von Stereotypen auch die Kompetenzfokussierung.

\subsection{Abgrenzung gegen andere AuBerungstypen}

Die formelhaften Charakterisierungen haben formal und funktional Ähnlichkeit mit einer Reihe anderer Äußerungstypen, von denen sie sich jeweils aufgrund bestimmter Merkmale unterscheiden und im Verhältnis zu denen häufig die Anwendung der Formulierungsverfahren am deutlichsten zu demonstrieren ist. Dazu gehören u.a. die im folgenden aufgeführten.

\subsubsection{Andere kondensierte Charakterisierungen}

Es gibt eine Reihe anderer Arten von kondensierten Charakterisierungen, die ebenfalls die Bekanntheit eines Bezugssachverhalts voraussetzen, wie

- Geschichtenkondensate, die sich durch die Mehrgliedrigkeit, ggf. auch durch Indexikalität und Redewiedergabe von den Charakterisierungsformeln unterscheiden; 
- historische Reminiszenzen, d.h. kurze Erinnerungen an historische Sachverhalte: der woll=doch niwwer fahre wu soin vadder gschdorwe is;

- kurze Zitate, z.B. von Aussprüchen der charakterisierten Personen; solche Zitate können die Kerninformation von Geschichtenkondensaten sein oder aus Witzen stammen; im Prinzip können auch Quasizitate in charakterisierender Funktion auftreten (vgl. Beitrag 2 und 7 ). In diesen Fällen ist die Form der Redewiedergabe das Unterscheidungsmerkmal.

\subsubsection{Andere Züge}

Typische reaktive Züge, die Ähnlichkeit mit formelhaften Charakterisierungen haben, sind:

(a) rhetorische Rückfragen wie die Äußerung von Frau Schumann am Beginn der schon weiter oben wiedergegebenen Sequenz kollektiven formelhaften Sprechens:

S. 2

20 HI : un der ihrn mann $\uparrow$ des=s auch

K : LACHEN

S. 3

$2 \mathrm{SU}: \quad \rightarrow \mathrm{a}^{\text {"ch }}$ die hat a noch=en mann

$4 \mathrm{HI}: 80^{=n}$ daggl $\downarrow$

(Text 2036/62)

Die Außerung von SU in 3,2 ist als eine witzige Bemerkung formuliert, bei der das gespielte Erstaunen (a"ch) eine stillschweigende gegenteilige Annahme signalisiert. Unabhängig davon, inwieweit SU in diesem Moment die Existenz von Otto präsent ist, trifft sie damit denselben Punkt wie die übrigen Sprecherinnen, nämlich die Irrelevanz von Otto, aber unter Verwendung eines anderen Formulierungsmusters, und zwar einer intonatorisch markierten Rückfrage.

(b) Reaktive Züge wie Identifizierungshilfen, mit denen Sprecher auf ein Referenzierungsproblem eines anderen Beteiligten reagieren:

- die wu iwwerall hiegeht

- die wu zweitausend marg schbendiert.

Diese unterscheiden sich von Charakterisierungsformeln durch das Relativsatzformat, die Akzentuierung der Kerninformation und die Intonation, die nicht die Aussagemodalität der selbstverständlichen Sicherheit ausdrückt. Es gibt aber eine gewisse Affinität $\mathrm{z}$ wischen ersten Identifizierungshilfen und Charakterisierungsformeln. Nach der Ökonomie der Bearbeitung von Identifizierungsproblemen sind erste Identifizierungshilfen tendenziell kurz; erst beim Scheitern der ersten, andeutungsweisen Problembearbeitung werden stufenweise expandiertere Hilfen angeboten. Darüber hinaus sind sehr viele erste Identifizierungshilfen formelnahe Formulierungen. Die Verwendung einer Kurzcharakterisierung 
als erste Identifizierungshilfe bietet die Möglichkeit, bei gelungener Identifizierung sofort mit formelhaftem Sprechen fortzufahren. ${ }^{21}$

\subsubsection{Themeneinführungen}

Vor allem wenn es sich um die Wieder-Thematisierung von geläufigen Gegenständen handelt, haben Themeneinführungen trotz ihrer initiativen Eigenschaften eine klare Affinität zu Charakterisierungsformeln. Sie verweisen in kurzer Form auf ein bekanntes thematisches Potential, z.B. eine charakteristische Eigenschaft einer bekannten Person:

- un der ihr mann des is au"ch so=n daggl

- die gehd aa in wirtschafte un trinkt.

Themeneinführungen sind aber mit der Einführung eines Referenten verbunden - das kontrastiert mit dem Kommentarformat -, und sie werden durch bestimmte Mittel markiert, welche die Relevanzhochstufung eines thematischen Potentials ausdrücken, und diese Eigenschaft kontrastiert mit der Aussagemodalität der Selbstverständlichkeit. Mittel sind z.B.:

(a) Aufmerksamkeitsappelle wie horsch emol, was määne se, awwer gu"gg die mo $\tilde{o}$. So werden mit den folgenden Äußerungen in ihrem jeweiligen Kontext eindeutig thematische Initiativen realisiert:

- was määne se wie dere Gerda ihr mudder noch gelebd hod was do los war;

- awwer gu"gg die mol o wie raffinierd so du"mm wie se is.

Im zweiten Beispiel wird mit dem Diskontinuitätssignal awwer ein thematischer Wechsel angezeigt, die Höreradresse wirkt als Aufmerksamkeitsappell, und die Kurzform einer Eigenschaftsdarstellung in allgemeinster Form gibt das thematische Potential an, das fokussiert werden soll; es folgt dann auch eine längere Auseinandersetzung über die "geschäftliche Raffinesse" von Gerda.

(b) Vergegenwärtigungsinstruktionen mittels der Partikeln doch und ja bei der Wiedereinführung von thematischen Potentialen, und zwar als Markierung von Bekanntheit ( $j a)$ zur Erinnerung bzw. als Markierung der Thematisierungsrelevanz (doch). So versucht z.B. eine Beteiligte im Gespräch mit der Charakterisierung von Otto durch der kriggd de eisschrongg abgschlosse an einer viel späteren Stelle nach mehreren Themenwechseln einen thematischen Rückgriff mit der arme Oddo hod doch ned on de eisschronk gederft. Diese Außerung unterscheidet sich von der inhaltlich entsprechenden früheren formelhaften Charakterisierung durch die explizite Nennung des Referenten (statt Proform), das Vergangenheitstempus (statt Präsens) und die Partikel doch.

(c) Lokalisierungen in bezug auf etablierte thematische Rahmen bei der Einführung (oder Wiedereinführung) von Teilthemen, z.B. durch auch. Das

21 Zur Struktur von Identifizierungssequenzen vgl. u.a. Kallmeyer (1977) und Kindt/Weingarten (1984). 
ist z.B. in un der ihr mann des is au"ch so=n daggl, der Fall. auch signalisiert die Subsumtion des Einzelfalles unter eine allgemeine soziale Kategorie "Dackel”; bei dieser subsumierenden Verwendung trägt auch regelmäßig den Satzakzent. Von der Kategorie "Dackel” war vorher nicht die Rede, sie gehört aber zum immer wieder aktualisierten thematischen Potential der Kategorisierung. Insofern handelt es sich hier um eine thematische Lokalisierung. An einer anderen Gesprächsstelle wird - ohne unmittelbaren Bezug auf den Nahkontext - ein weiteres Merkmal von Gerdas Lebenswandel als Teilthema eingeführt: die gehd aa in werddschafte un trinkt. Die Partikel aa (=auch) trägt bei dieser Verwendung als Indikator für die Auflistung von Eigenschaften keinen Akzent. Auch in diesem Fall wird durch auch die Wiederaufnahme eines thematischen Potentials angezeigt.

\section{Formelhaftes Sprechen im Tratsch: „Der arme Otto”}

Im folgenden soll ein längerer Ausschnitt aus einem typischen Tratschereignis in der Gruppe der Filsbachfrauen analysiert werden. Aus diesem Gesprächsabschnitt stammen die meisten bisher zitierten formelhaften Charakterisierungen. Gesprochen wird über ein Tratschobjekt, das in der Folge der Gruppentreffen immer wieder aufgegriffen wird: Gerda, eine im Stadtviertel allgemein bekannte ältere Frau, und ihr Mann Otto. Gerda ist eine trinkfeste und äußerst gesellige Person, die als „Königin” des Viertels bezeichnet wird und sich gerne in dieser Rolle sieht. Das Verhältnis zwischen ihr und Otto ist das eines hausdrachens zu einem hampelmann (vgl. die Analyse der sozialen Kategorisierung im folgenden Beitrag). Die prominente Figur ist Gerda, während Otto nur im Verhältnis zu ihr als Tratschobjekt interessant ist. Dieser Relevanzeinstufung entspricht die normale Thematisierungsfolge: es wird eine Weile über Gerda geredet, und dann taucht das Zusatzthema "Otto” auf. In der ausgewählten Sequenz geht es um dieses Zusatzthema.

Der Ausschnitt gibt einen zusammenhängenden Bogen der Themenbehandlung wieder mit einer charakteristischen Abfolge von Thematisierungsformen. In den verschiedenen Behandlungsphasen erscheinen unterschiedlich explizite und expandierte bzw. kondensierte Darstellungsformen zum Thema, d.h. kurze Erwähnungen, detaillierte Erzählungen usw., und im Zusammenhang damit unterschiedlich formelhafte ÄuBerungen und unterschiedliche Arten von Formeln.

Die Analyse konzentriert sich auf die folgenden Punkte:

- Auftreten und Entwicklung von Formelhaftigkeit im Gespräch

- Sequenzmuster für gemeinsames formelhaftes Sprechen

- unterschiedliche Interaktionsmodalitäten in Verbindung mit formelhaftem Sprechen. 


\subsection{Themeneinführung und kondensierte erste Behandlung}

Der erste größere Gesprächsabschnitt gliedert sich in vier Teilstücke, und zwar die erste Themeneinführung (a), eine Identifizierungssequenz (b), eine Runde formelhafter Charakterisierungen (c) und eine Relevanzhochstufung des Themas als Auslöser für eine weitergehende Behandlung (d).

(a) Erste Einführung des Themas "Otto"

Frau Zimmermann (ZI) erzählt eine Geschichte über Gerda (wie Gerda in einem Café Hof hält und Freundinnen zum Trinken einlädt). Kommentierend charakterisiert eine andere Beteiligte Gerda als hausdrache. Ingrid (IN) hat ein Verstehensproblem: Sie bezieht die Charakterisierung hausdrache versuchsweise auf Otto, der zuletzt erwähnt wird in einem Gerda-Zitat (moin alde). Das Verstehensproblem wird von den anderen nicht bearbeitet, sondern Frau Zimmermann als nächste Sprecherin reagiert mit einer Charakterisierung von Otto (der hod alles schaffe misse $\downarrow$ ohje ohje $\downarrow$ 1,10). Mit der Identifizierungsfrage von IN zur Person von Gerda $(1,12)$ ist die Behandlung des Themas "Otto" zunächst einmal wieder zu Ende.

S. 1

$1 \mathrm{ZI}:(\ldots)$ un no hod se noch drei * ä:hm * ä:h ki"rsch/ nix

2 ZI: kirsch * vassa bschdelld un so * >na havg=isch gsa vie

$3 \mathrm{ZI}: \mathrm{geh}=\mathrm{da}=\mathrm{s} \uparrow$ seschd se jo moin alde $\underline{(\ldots)}$

$4 \mathrm{SU}$ :

5 MÜ:

>hausdrache

6 IN :

ihrn mannl

(1):

$7 \mathrm{ZI}$ :

8 мü: :

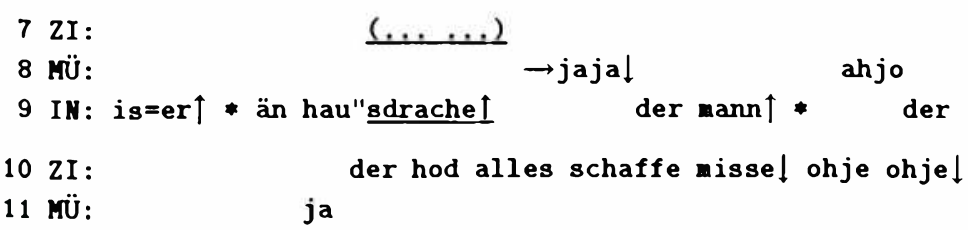

9 IN : is=er $\uparrow$ * än hau"sdrache $\uparrow$

der hod alles schaffe misse $\downarrow$ ohje ohje $\downarrow$ ja

$10 \mathrm{ZI}$ :

11 MÜ:

12 IN : ehemann $\uparrow \mathrm{ja}$

$13 \mathrm{ZI}$ :

$14 \mathrm{KU}$ : ja:" $<j a: "$ ja

15 IN: des die fraa die do in H5 vohnt $\uparrow$ $\mathrm{K}$ :

DURCHEINANDER

16 2I :

ja:

$17 \mathrm{KU}:$

18 IN : film $\mid$ äh/

$\mathrm{K}$ :

LACHEN 
Der Ausdruck alles schaffen müssen ist eine feste Formel für die Eherolle des sog. „Hampelmanns”, d.h. des sich unterwerfenden Partners eines „Hausdrachens”. In dieser Verwendung bedeutet die Formel „die ganze Hausarbeit erledigen müssen” bzw. noch allgemeiner „die rollengebundenen Aufgaben der Frau erledigen müssen". Das ist eine definierende Eigenschaft der Kategorie „Hampelmann”: Wenn jemand als „Hampelmann” kategorisiert wird, kann man erwarten, daß er "alles schaffen muB”. Insofern handelt es sich bei der Verwendung des Ausdrucks hier um eine ganz allgemeine, an der Definition sozialer Kategorien orientierte Charakterisierung. Die Formel indiziert eine soziale Kategorie; ihre Verwendung setzt keine spezielle Kenntnis der Realisierungsformen der Hausdrachenehe im vorliegenden Fall voraus. ${ }^{22}$

Erklärungsbedürftig ist das Vergangenheitstempus in der formelhaften Charakterisierung. Es ist nicht durch die Referenz auf eine vergangene Welt motiviert; Gerda und Otto gehören zur „Filsbachwelt”. Vielmehr handelt es sich um eine kontextuelle Anpassung, die wesentlich gesprächsorganisatorisch bedingt ist. Frau Zimmermann berücksichtigt in ihrer Äußerung zwei Kontexte: zum einen beantwortet sie die Frage von Ingrid (die ggf. gar nicht speziell an sie adressiert war), zum anderen tut sie dies - durch die Verwendung des Perfekts - im indexikalen Rahmen ihrer Erzählung, die gerade gestört worden ist. D.h., sie beantwortet die Frage wie eine zu ihrer Erzählung gestellte Frage: „Während Gerda im Café Hof gehalten hat, war er zu Hause bei der Hausarbeit"; das gehört im Zweifelsfall nicht zur Geschichte, entspricht jedoch dem typisierten Bild von Gerda und Otto (das kann gar nicht anders sein und ist deshalb jederzeit zu interpolieren). Mit dieser Bezugnahme auf den indexikalen Rahmen der Erzählung führt Frau Zimmermann die an dieser Stelle divergierenden Gesprächsaktivitäten wieder zusammen.

Für Eingeweihte ist die Bedeutung des formelhaften Ausdrucks sofort klar, für Außenstehende (wie Ingrid) ist der Quantor alles nicht ohne weiteres interpretierbar. Außenstehende können eine relativ unspezifische und unsichere Bedeutung zuschreiben im Sinne von 'Rechte und Pflichten sind ungleich verteilt,

22 Diese kurze Sequenz der Charakterisierung von Gerda und Otto wird im nächsten Beitrag noch genauer analysiert im Hinblick auf die Kategorisierung. Die beschriebene Verwendungsweise der Charakterisierung heißt nicht, daß es keine konkreten Hintergründe geben würde. Über ein Jahr später wird über Ottos Haushaltspflichten z.B. so geredet:

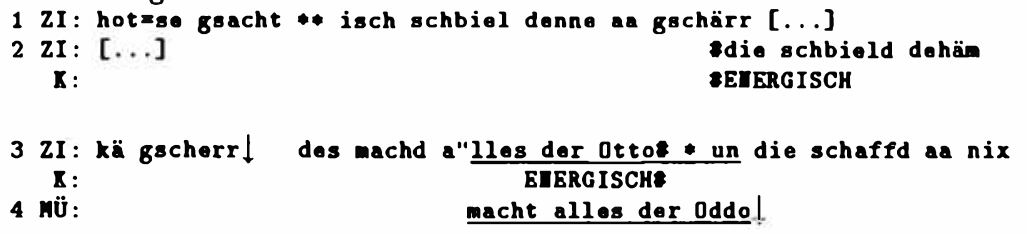

5 ZI: die hod ihr fingernägel tut sisch pflege $\downarrow$ machd alles der 0tto 
und Otto ist der Benachteiligte'. Eine solche vage Interpretation ist textuell erschließbar, eine Spezifizierung der Bedeutung ist aber ohne Kenntnis des formelhaften Ausdrucks nicht möglich. In einer Verwendung wie hier, d.h. in einer kooperativen Situation mit einem akzeptierten Wissensdefizit eines Gesprächspartners kann eine solche Außerung eines Eingeweihten dem Außenstehenden gegenüber immer als Andeutung eines Charakterisierungspotentials fungieren, das durch Nachfragen und Interessebekundungen abzurufen ist. ${ }^{23}$

\section{(b) Identifizierung als Voraussetzungssicherung}

Ingrid geht auf das von Frau Zimmermann angedeutete Charakterisierungspotential nicht ein, weil sie noch mit Verstehensproblemen beschäftigt ist, die zur Voraussetzungssicherung für die weitere Interaktion bearbeitet werden müssen. Es geht um die Identifikation von Gerda auf der Grundlage des schon vorhandenen Wissens: is des die fraa die do in ha fünf wohnt $\uparrow$ die in dem film $\uparrow$. Es gibt einen Fernsehfilm über den Stadtteil mit Aufnahmen einer Gruppe von Frauen aus der Stammbevölkerung, darunter Gerda; diese hatte sich für die Gelegenheit herausgeputzt und ihren Schmuck angelegt. Den Film kennen alle. Die Rückfrage von Ingrid löst eine Sequenz der Voraussetzungssicherung aus:

S. 1

12 IN : is

$13 \mathrm{ZI}$ :

$14 \mathrm{KU}$ : ja:" ${ }^{\text {jo:" }}{ }^{\text {ja }}$

15 IN : des die fraa die do in $\mathrm{H} 5$ vohnt $\uparrow$ K : die in dem

DURCHEINANDER

$16 \mathrm{ZI}$ :

ja:

$17 \mathrm{kU}: \quad \mathrm{ja}: \downarrow$ die vu do ihr/ ihr/ ihr/ ihr brilljonde do

18 IN : $f$ ilm $\uparrow$ äh/

$\mathrm{K}$ :

\section{LACHEN}

$19 \mathrm{KU}: \underline{(\ldots)}$

$20 \mathrm{SU}$ : $<$ die

21 IN : aaah >des is die do $\uparrow *$ un die heißt Gerddl $\downarrow$

22 ZI: *Ge"rda \#

$\mathrm{K}$ : *GEDEHNT\#

$23 \mathrm{SU}$ : brü" 11 jande

${ }^{23}$ Ein solches Charakterisierungspotential verdeutlicht z.B. Frau Zimmermann, als Maria einer dritten Beteiligten gegenüber eine Identifizierungshilfe der geschilderten Art gibt:

1 MA: die kenne sie net $\uparrow$ die oo ivoeral hiegehd

2 2I:

oos gas gibt!

3 ZI: hod der parrer vas $\uparrow$ is=se a do $\downarrow$ 
$24 \mathrm{KU}$ :

ja: Gerdda

$25 \mathrm{HH}$ :

der

26 IN :

Sun die heiBt Gerddl

S. 2

$1 \mathrm{ZI}$ :

Gerda

2 Wh: krigg=de eisschrongg abgschlosse daß=a ned zuviel fribd $\downarrow$

3 мÜ:

Gerda

Die Klärung ist für Ingrid einmal im allgemeinen Sinne der Voraussetzungssicherung wichtig, zum anderen sicher auch im Hinblick auf ihr Beobachtungsinteresse - sie ist auf eine ethnographische "Ader” gestoßen: eine außergewöhnliche Figur, vielleicht ein Original. Ingrid stellt sich durch ihre Aktivitäten als Lernende dar. Charakteristisch dafür sind einerseits gehäufte Rückfragen (vgl. $1,15 ; 1,18 ; 1,21 ; 1,26)$, mit denen sie ggf. auch den Aktivitätsfluß unterbricht, und die manifeste Darstellung von Verstehensleistungen, u.a. durch Wiederholung und explizite Verknüpfung von Informationen (>des is die do $\uparrow$ un die heißt Gerddl $\downarrow$ ). Ingrids Intervention wird von mehreren Gruppenmitgliedern sehr kooperativ behandelt. Insofern wird von den Beteiligten Ingrid interaktiv der Status einer „Novizin” zugeschrieben.

Die Identifizierungshilfen werden zur sozialen Symbolisierung benutzt, d.h., die Orientierung auf das thematische Potential und die von ZI initiierte Interaktionsmodalität werden aufrechterhalten. Die Identifizierungshilfe $j a: \downarrow$ die $w u$ do $i h r / i h r / i h r / i h r$ brilljonde do (1,17f.) zeigt die Anwendung der Verfahren von Hervorhebung und Zuspitzung zusammen mit der Kondensierung: die manifeste Wortsuche (ihr/ihr/ihr/ihr) läßt die Anwendung besonderer Kriterien vermuten, nach denen nicht jede als Identifizierungshilfe mögliche Formulierung akzeptabel ist. Wenn brilljonde (als nicht-erstbester Formulierungskandidat) ausgewählt worden ist, dann im Sinne der Zuspitzung auf „sprechende Details". So wird der Ausdruck jedenfalls von Frau Schumann (SU) behandelt: ihre Folgeäußerung die brü"lljande $(1,23)$ wird mit den Merkmalen der falschen Vornehmheit artikuliert (vgl. vorigen Beitrag). Die Reformulierung von Frau Schumann intensiviert die Symbolisierung des „königlichen” Auftretens von Gerda.

\section{(c) Charakterisierungsrunde als kondensierte Themenbehandlung}

Frau Wichmer (WH) kommt auf das Thema „Otto" zurück; sie behandelt die erste formelhafte Äußerung von Frau Zimmermann $(1,10 \mathrm{ff}$.) als AnlaB für weitere Charakterisierungsaktivitäten. Es kommt eine Sequenz formelhafter Charakterisierungen in Gang, bei der Frau Wichmer und Frau Zimmermann als Protagonistinnen fungieren und die übrigen Beteiligten mit kurzen Reaktionen des Erstaunens (Frau Schumann) und der wissenden Zustimmung (Frau Müller - MÜ) gleichsam als Chor. Die Unterschiede der Reaktionen hängen damit zusammen, daB Frau Müller Filsbacherin ist und die lokale Welt und ihre 
Geschichte kennt, während Frau Schumann in Mannheim eine „Neubürgerin” und in der Gruppe eine später Hinzugekommene ist und noch wenig Hintergrundwissen über die Filsbachwelt hat.

S. 1

$24 \mathrm{KU}:$

ja: Gerdda

25 Wh:

26 IN : $\quad$ sun die heiBt Gerddl

der

S. 2

$1 \mathrm{ZI}$ : Gerda

2 WH: krigg=de eisschrongg abgschlosse daB=a ned zuviel fribd $\downarrow$

3 мÜ:

Gerda

$4 \mathrm{ZI}: \quad(\ldots)$

5 SU: LACHT ERSTAUNT

$6 \mathrm{KU}$ :

$(\ldots)$

7 WH:

$\rightarrow$ (oh dafsa ned soviel friBd)

8 мÜ:

$9 \mathrm{ZI}$ :

der

$10 \mathrm{SU}$ :

taach*

$\mathbf{K}:$

*ERSTAUNT

$11 \mathrm{HH}: \leftarrow$ der kriggd de eisschrongg abgschlosse $\downarrow$

$12 \mathrm{ZI}:$ kriggd=n abgschlosse $\downarrow$ ja un/

$13 \mathrm{NH}$ :

14 MÜ:

jaja * jaja * un venn=a brav is

ja:"

15 WH: kriggd=a halvi dafel schoklad $\downarrow$ ve"he venn=a mehr vill $\downarrow$

16 MÜ:

ouu

$\mathbf{R}$ :

DURCHEIMANDER

17 ZI : Ł-un alle schdund ei"ne zigaredd

18 HE: jaja

19 แH :

isch hab=m imma paar

20 IN :

va:s†

$21 \mathrm{WH}$ : gevve ach gott der=8 arbeidslos】

22 Mü: der arbeided nix

$23 \mathrm{KR}$ :

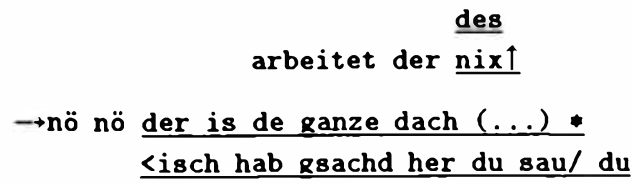

24 IN :

252 I : sisch hab gsachd her du sau/du

$26 \mathrm{HH}$ :

$27 \mathrm{KR}$ : 1 äß $=$ der sich gfallen $\uparrow$

$28 \mathrm{ZI}:(\ldots)$

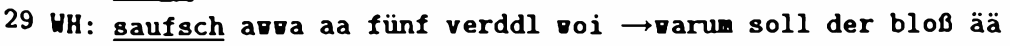


$30 \mathrm{KU}:$

31 UH: zigaredd raache in de schdunnd $\uparrow$ LACHT

$32 \mathrm{KU}$ : vare mol fordd >var die * fra Kaddovitz debei * un do hod er

Sequenzverlauf:

- Anknüpfung und Etablierung der Formelhaftigkeit: Mit der eindeutig formelhaften Charakterisierung der krigg=de eisschrongg abgschlosse knüpft Frau Wichmer an das Thema "Otto" an. Sie setzt an der ersten Position nach dem eindeutigen AbschluB der Identifizierungssequenz mit ihrer Äußerung ein. Damit liefert ihr Kommentar entsprechend den dargestellten Verwendungsregeln (vgl. Kap. 3.3.) eine Fortsetzung mit einer unmittelbaren Reaktion. In der sequenziellen Entwicklung von der ersten Charakterisierung (der hod alles schaffe misse $\downarrow ; 1,10$ ) zu den Formulierungen im Präsens (ab Z. 1,25) liegt eine Steigerung der Formelhaftigkeit.

- Definitive Etablierung der Interaktionsmodalität der fraglosen Sicherheit: Die formelhafte Charakterisierung ist expandiert durch eine Begründung ( $d a \beta=a$ ned zuviel fri $\beta d \downarrow)$, d.h. hat nicht das minimale Format eines Kurzkommentars. Diese Begründung wird in Reaktion auf das Erstaunen von Frau Schumann durch Wiederholung bekräftigt. Die Expansion ist im Prinzip gegenläufig zur Entwicklung des formelhaften Sprechens, das aber nach der Bestätigung von Frau Müller mit der Wiederholung der Kurzcharakterisierung wieder verstärkt wird $(2,7)$. Gegenüber der in einem Bogen gesprochenen zweiteiligen Formulierung mit Begründung stellt die Wiederholung nur der Kurzcharakterisierung in einem neuen Sprecheinsatz eine Kondensierung dar. Frau Zimmermann kondensiert in ihrer Reformulierung weiter $(2,9 / 12)$. Diese Folge von verkürzenden Reformulierungen unterschiedlicher Sprecherinnen etabliert endgültig die für das formelhafte Sprechen charakteristische Interaktionsmodalität.

- Austausch formelhafter Charakterisierungen als gemeinsame Produktion im Wechselspiel: Auf eine Charakterisierung und eine erste bestätigende bzw. honorierende Reaktion (Lachen, ja:) folgt jeweils eine korrespondierende Charakterisierung. Dabei ist im Prinzip Frau Wichmer initiativ und Frau Zimmermann folgt, aber in 2,12 zeigt der Formulierungsansatz von Frau Zimmermann, daB auch sie bereits zur Fortsetzung ansetzt. Diese Äußerungen weisen eine hohe Korrespondenz untereinander auf in bezug auf Formulierungsübernahmen (der kriggd abgschlosse), parallele Konstruktion bzw. Konstruktionsübernahme („er kriegt”, d.h. Otto als Patiens), semantische Parallelität (halwi dafel schoklad - alle schdund ei"ne zigaredd) sowie korrespondierende Prosodie (relativ schnell gesprochen, die einzelnen Charakterisierungen „in einem Bogen”, ohne große Tonhöhenschwankungen und mit leicht fallender Intonation am Schluß). Der gemeinsame semantische Rahmen ist die in der ersten Charakterisierung von Frau Wichmer ausgedrückte Idee des von Gerda erzwungenen Konsumverzichts Ottos; alle ÄuBerungen dieser Sequenz formulieren Variationen dieses Themas. Inner- 
halb der Sequenz gibt es - im Rahmen der Formelhaftigkeit - kleine Expansionen und Expressivitätssteigerungen wie in un wenn=a brav is kriggd=a halwi dafel schoklad $\downarrow$ we"he wenn=a mehr will $(2,13 / 15)$. Solche Expansionen und Hervorhebungen (wie die von we"he) sind Andeutungen szenischer Gestaltung ebenso wie eine leichte Sprachverschiebung in Richtung Standard in un alle schdund eine zigaredd, mit welcher der autoritäre Charakter von Gerdas Sprechen symbolisiert wird.

- Andeutung von Hintergrundgeschichten als Initiative zum Wechsel der Interaktionsmodalität: Die Äußerung isch $h a b=m$ imma paar gewwe $(2,19 / 21)$ von Frau Wichmer ist syntaktisch nicht mehr parallel zu den voraufgehenden (,ich" statt „er" in Subjektposition), historisch indexikal - wenn auch empirisch generalisierend - und verschiebt das Thema zu "Ottos Bedürftigkeit”. Diese Äußerung initiiert einen Modalitätswechsel.

- Fortsetzung der Interaktionsmodalität der Formelhaftigkeit bei der Behandlung des veränderten Themas: Rückkehr zum Präsens und zur Kommentarkonstruktion. Auch hier zeigen sich sofort Korrespondenzphänomene in der Behandlung durch die Gesprächspartner: Frau Wichmer startet fast gleichzeitig mit der $=s$ arbeidslos $\downarrow(2,21)$ mit der inhaltlich identischen Charakterisierung von Frau Müller der arbeided nix $\downarrow(2,22)$. Auch diese Charakterisierung ist metaphorisch zu verstehen: Otto ist Rentner, er bekommt eine relativ gute Rente; alle Beteiligten wissen das und reden wiederholt darüber. arbeitslos wird als Ausdruck für eine sehr negativ bewertete Bedürftigkeit verwendet; ein Mann, der nicht arbeitet, ist ein wertloser Mann (vgl. den nächsten Beitrag).

- Interessensbekundung der Externen Ingrid und Frau Kranz an einer ernsthaften, auf jeden Fall für sie informativen Themenbehandlung: Sie stufen die Relevanz des Themas hoch. Ihre bewertenden Reaktionen wa:s $\uparrow(2,20)$ und des läß $\beta=$ der sich gfallen $\uparrow(2,23 / 27)$ geben zwei Impulse für die weitere Behandlung: Sie manifestieren ihren Plausibilisierungsbedarf (im Sinne von „ist das wirklich so?” bzw. „sowas gibt es?”) und eine Bewertung von Ottos Verhalten.

- Übergang zu einer Hintergrundgeschichte: Frau Wichmer formuliert die historisch indexikale Darstellung eines Erfahrungshintergrundes im Format eines Geschichtenkondensats: <isch hab gsachd her du sau/du saufsch awwa aa fünf verddl woi $\rightarrow$ warum soll der bloß äa zigaredd raache in de schdunn $\uparrow$ (2,26-2,31; es geht um ein Gespräch zwischen Frau Wichner und Gerda über Otto). Der historisch-indexikale Charakter der Darstellung verbindet sich mit der Referenz auf singuläre Ereignisse. Damit ist die Formulierungsweise der sich anschließenden Erzählungen erreicht. Frau Wichmer setzt also ihre Linie des Modalitätswechsels fort. Auch daß sie eine spezifische Betroffenheit zeigt, kontrastiert mit der Modalität der selbstverständlichen Sicherheit beim formelhaften Sprechen: Sie ergreift Partei für Otto. Mit ihren beiden 
letzten Äußerungen signalisiert Frau Wichmer Widersprüche zu den in den Formeln dargestellten Urteilen. Sie zeigt, daß es für sie auf einer anderen Ebene der Themenbehandlung noch Eigenschaften von Otto gibt, die nicht unter die voraufgehende einfache Kategorisierung zu subsumieren sind.

Die in der Sequenz gemeinsamen formelhaften Sprechens formulierten Charakterisierungen sind inhaltlich spezifischer als die auslösende Äußerung von Frau Zimmermann, die eine kategoriengebundene Eigenschaft formulierte. In der Entfaltung des formelhaften Sprechens verbinden sich kategoriengebundene Eigenschaften und Aktivitäten mit einer fallspezifischen Konkretisierung. Kategoriengebunden ist, daB die Kernidee von der Entmachtung des Mannes in der Beziehung zwischen „Hausdrache” und „Hampelmann” mit der Übertragung des Erziehungsverhältnisses Mutter-Kind auf das Verhältnis Frau-Mann verbunden wird. Die ÄuBerung un wenn=a brav is kriggd=a halwi dafel schoklad we he wenn=a mehr will $\downarrow(2,13 / 15)$ formuliert Kernelemente eines Erziehungssystems, und zwar Belohnung bzw. Versprechen von Belohnung (Schokolade als Belohnung für "Brav-sein") und Bestrafung bzw. Androhung von Strafe (we"he wenn=a mehr will $\downarrow$ ). Für das Wohlverhalten als Unterwerfung fungiert brav als Schlüsselwort. Es erscheint vielfach in diesem Sinne und indiziert, angewendet auf Ehemänner, stets ein Verhältnis zwischen Hausdrachen und ihren unterworfenen Männern. Insofern gehört „Brav-sein” zu den kategoriengebundenen Aktivitäten und Eigenschaften von „Hampelmännern”. Dieses allgemeine Konzept der strengen Erziehung des Mannes als Merkmal des Hausdrachens wird hier mit der fallspezifischen Kenntnis verbunden, daB bei Gerda und Otto der erzwungene Konsumverzicht Ottos ein zentrales Merkmal ihrer Ehebeziehung ist.

Gegenstand des gemeinsamen formelhaften Sprechens in der Sequenz paralleler Charakterisierungen ist also die Auffüllung einer allgemeinen Kategorie mit Fallelementen, die wiederum als typische behandelt werden. Die apodiktische Generalisierung ist dabei ein Indikator für Eigenschaften, die als kategoriengebunden eingestuft werden. Durch die Ausgestaltung wird zwar eine reichere Typisierung geschaffen, diese bleibt aber auf eine zugrundeliegende einfache Kategorisierung bezogen.

\subsection{Konkretisierung und Detaillierung durch eine Erzählung}

Frau Wichmers Andeutung eines Problematisierungspotentials hat an dieser Gesprächsstelle keinen Erfolg; stattdessen folgt eine Erzählung von Frau Kunz ( mir ware mol fordd >), welche einen weiteren Aspekt von Ottos unfreiwilligem Konsumverzicht darstellt und insofern den thematischen Rahmen der formelhaften Charakterisierungen unmittelbar fortsetzt, jetzt aber auf der Ebene der detaillierten, explizit indexikalen Darstellung. 
S. 2

$30 \mathrm{KU}$ :

LACHT

$31 \mathrm{HH}$ :

$32 \mathrm{KU}$ : vare mol fordd > var die * fra Kaddovitz debei * un do hod er

S. 3

$1 \mathrm{ZI}$ :

$2 \mathrm{KU}:$ sisch vas beschde"lle volle $\uparrow$ do"/ vas veeb isch $\uparrow$

$\mathrm{K}$ :

$(\ldots .$.

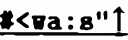

\#REIFEND

3 BO:

isch

$4 \mathrm{ZI}: \quad$ der derf doch nixl

$5 \mathrm{KU}:$ hodse $\uparrow \quad$ ga"s $\uparrow \rightarrow$ hod se gsacht $\uparrow$ isch glaab

$\mathrm{K}:$

6 BO: var debei

$7 \mathrm{KU}:$ daß du verriggd bisch $\uparrow$ do geh hään un mach doi (..)* avva $\mathrm{K}$ :

KEIFEND\#

$8 \mathrm{ZI}$ :

jaja

$9 \mathrm{KU}:$ sie" hod avga gfresse $=3$ deierschde

10 мÜ: $\quad$ oooooh $\leftarrow d u: " k r i g g s c h / d u$

$11 \mathrm{HE}$ :

$12 \mathrm{KU}: \quad$ ha"ggbro:de mit $(\ldots \ldots \ldots \ldots)$

13 MÜ: kriggsch äh:/

14 WH: hagg/ nää: eier eier- **ier

$15 \mathrm{KU}: \quad \rightarrow$ domols hod=a haggbrode griggd des va=s

$16 \mathrm{WH}$ : mid schingge

$17 \mathrm{KU}$ : billigschde bloß fünf maag gekoschd

$18 \mathrm{HH}$ : eier mid schingge

$19 \mathrm{KU}$ : onner hod vas gfresse des/ des hod fuchzehne gekoschl *

$20 \mathrm{WH}$ : griggd=a meischdens |

$21 \mathrm{SU}: \geq$ aach<

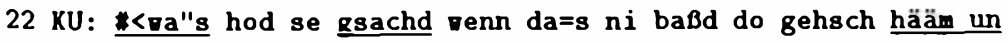

$\mathrm{K}:$ *SCHARF, HOCH

23 MÜ:

>oun<

24 IN :

LACHT

$25 \mathrm{ZI}$ :

(isch glaab)

$26 \mathrm{KU}:$ kochsch da ras* * ooouuu

var der bra:v *

K: SCHARF, HOCH\#

27 พH:

<schri"m ivver de ozea:n-

28 IN : LACHT 
S. 4
121 :
ia ja $(\ldots \ldots)$
nänä
$2 \mathrm{HE}$ :
nänä
$3 \mathrm{kU}:$ der hod schä: gessel
4 HH:
schvi"minger de ozeanl*
$\mathrm{X}$ : DURCHEIMANDER

Die Erzählung enthält eine Kernszene mit Gerda und Otto als Beteiligten und mit Gerda als zitierter Sprecherin. Inhaltlich entspricht die Szene einer geläufigen Charakterisierungsformel, wonach Otto beim Essen in der Gaststätte kurz gehalten wird, während Gerda ausgiebig tafelt; diese Formel erscheint in einigen Varianten, z.B. er kriggd nudle mid schingge, sie frißt zwee schdeks oder er kriggd eier mid schingge .... Die Erzählerin hat hier nun eine kurze Formulierungsschwierigkeit (äh:/ ha"ggbro:de, 3,10ff.). Frau Wichmer beteiligt sich zunächst mit einer Bestätigung der gefundenen Formulierung, bricht aber sofort ab und korrigiert zugleich sich selbst und die Erzählerin, die damit unterbrochen wird. In der Korrektur wird die stereotypisierte Normalform der Szene reproduziert (nää: eier...eier eier mid schingge, 3,14/16). Eine weitere Zuhörerin stimmt sofort ein (HE: eier 3,14). Die Unterbrechung der narrativen Äußerung mit einer so manifesten Korrektur (nää:) zeigt an, wie präsent den Zuhörerinnen die Normalform des Ereignisses und die dazugehörige sprachliche Formel sind (eier mid schingge).

Die Unterbrechung durch eine manifeste Fremdkorrektur ist nach den konversationsanalytisch gefundenen Präferenzregeln für Korrekturen, nach denen Selbstkorrekturen systematisch der Vorzug gegeben wird, auffälig (vgl. Schegloff/Jefferson/Sacks 1977); und offensichtlich ist sie auch für die Beteiligten behandlungsbedürftig: Es findet eine Renormalisierung statt, in deren Verlauf beide Versionen gerechtfertigt werden. Die Erzählerin rechtfertigt ihre Version durch die historische Wahrheit:

$\rightarrow$ domols hodxa haggbrode griggd

des va=s billigschde $*$ bloß fünf maag gekoschd [...]

un die onner hod vas gfresse

des/ des hod fuchzehne gekosch $\downarrow(3,15 / 19)$.

Dabei weist sie zugleich nach, daß ihre Darstellung mit der stereotypen Inhaltsfigur voll übereinstimmt. Frau Wichmer rechtfertigt den Eingriff durch eine empirische Generalisierung als Grundlage der stereotypen Figur:

eier mid schingge griggd=a meischdens $(3,18 / 20)$. 
Der Kern der stereotypen Charakterisierung, die krasse Ungleichbehandlung, ist allen vertraut und völlig unstrittig, die Divergenz betrifft nur die Auswahl der „sprechenden Details” für ihre formelhafte Verdeutlichung. ${ }^{24}$

Insgesamt bleibt bei dieser Erzählung und ihrer Rezeption die Ausrichtung auf gesicherte Typisierungen, wie sie für die formelhaften Charakterisierungen ausschlaggebend sind, erhalten, auch wenn sich das Detaillierungsniveau ändert.

\subsection{Thematische Ausweitung}

In Gesprächen, in denen eine Präferenz für Erzählen gilt ${ }^{25}$, folgen in der Regel auf erfolgreiche Erzählungen Reaktionen in Form von Kommentaren sowie ergänzende und analoge Darstellungen, häufig weitere Erzählungen. Dabei wird der thematische Rahmen der Erzählung zunächst beibehalten bzw. nur allmählich verschoben. Auch Frau Wichmer versucht eine solche Fortsetzung mit schwi"mm iwwer de ozea:n\, die aber erfolglos bleibt, wie an der mangelnden Beteiligung der anderen Anwesenden zu sehen ist. Im Unterschied dazu hat die Fortsetzungsinitiative von Frau Held (HE) der woll=doch niwwerfahre Erfolg.

S. 4

$1 \mathrm{ZI}$

ja ja $(\ldots \ldots)$

nänä

2 HE:

$3 \mathrm{KU}:$ der hod schä: gessel

4 HH: Schvi"mm iover de ozean】 *

$\mathrm{K}$ : DURCHEINANDER

5 WH:

der=s doch vun Ameriga- schvi"mm ivver

6 MÜ: >do hod=a=s

(besser)

(.................

$7 \mathrm{HE}:$

ach * der volld/

Sder poll=doch

$8 \mathrm{KU}:$ die hod=n schä zommegschisse $\uparrow$

9 WH: de ozean $\downarrow$ LACHT

$10 \mathrm{ZI}$ : sach/ hot se=n ne

11 HE: nivverfahre vu soin vadder gschdorve is nid

$12 \mathrm{ZI}$ : gelobt nä näl.

$13 \mathrm{HE}:$ die hod=n ni"schd geloBd $\downarrow$

$14 \mathrm{HH}$ : $\mathrm{K}$ : $>$ der vär vielleisch *<der vär nimmer *HOCH

$15 \mathrm{KR}$ : $\underline{\text { a"ch }}$

24 Bei der Präferenz von Frau Wichmer für die Formulierung eier mid schingge spielt u.U. auch die größere rhythmische Geschlossenheit eine Rolle; vgl. auch Coulmas (1981a).

25 Erzählen in Situationen ohne diese Präferenz für das Erzāhlen wird in Beitrag 6 untersucht. 
$16 \mathrm{ZI}$ : ah der vär nimer kumel

17 HE: garnimmer kumel de"r vär nimer huneel

18 UH: ku"net*

$\mathrm{R}: \quad$ :

19 หÜ: $\quad$ der vär niner

$20 \mathrm{2I}: \quad \leftarrow$ ava: trotzde:n $\rightarrow$ ah denn gherd/ der ou=s/ dem

21 MÜ: kunne

22 ME:

Die ÄuBerung schwimm iwwer de ozean $\downarrow$ ist inhaltlich parallel zu der VorgängeräuBerung (do gehsch hääm un kochsch da was, 3,22/26), sie formuliert ebenfalls die Inhaltsfigur "herzlos wegschicken”. Die Formulierung in der zweiten Person ist offenkundig ein Zitat, das mit einer Art Singsang realisiert wird und sich dadurch auch intonatorisch von den formelhaften Kurzcharakterisierungen deutlich unterscheidet.

Trotz der expliziten Remotivierung der=s doch vun Ameriga und der Wiederholung des Zitats $(4,5 / 9)$ erhält die Sprecherin aber keine angemessene Reaktion. In der Folge wird zwar das neue thematische Element 'Otto ist aus Amerika' aufgenommen, aber in einem Sinne, welcher der inhaltlichen Figur ( Otto herzlos wegschicken”) entgegenläuft ("Otto darf nicht weg”, 4,11ff.). Für das Scheitern von Frau Wichmers ÄuBerung gibt es vermutlich zwei Gründe. Zum einen fehlen zu diesem Zeitpunkt den übrigen Teilnehmern die notwendigen Hintergrundinformationen. Es handelt sich auch bei dieser ÄuBerung um das Zitat einer Gerda-ÄuBerung gegenüber Otto; die übrigen Anwesenden kennen aber diese Geschichte noch nicht. Zum anderen spricht einiges dafür, daB den Gruppenmitgliedern diese Art des formelhaften Sprechens als charakterisierender Kommentar im Tratsch nicht geläufig ist. Die Formulierungsweise kollidiert mit den vertrauten Formulierungsmustern.

Die FolgeäuBerung von Frau Held, die das Thema „Amerika” aufnimmt, wird durch die Verwendung eines Thematisierungsindikators doch als thematische Initiative markiert:

<der voll=doch nivierfahre wu soin vadder gschdorve is $(4,7 / 11)$.

Die historische Reminiszenz wird von der Sprecherin mit ihrer Fortsetzung zu einem dreigliedrigen Geschichtenkondensat vervollständigt:

die hod=n ni"schd geloßd $\downarrow$ * der vär vielleisch

garnimer kumel $\downarrow(4,13 / 16)$

Die Formulierung ist historisch-indexikal und singulär, aber zumindest ist sie nicht als Zitat markiert, sondern vollzieht schon die Überführung von szenischer Darstellung mit Redewiedergabe in eine Formulierung in der dritten Person.

Mit ihren Reaktionen zeigen die übrigen Gruppenmitglieder, daß es sich um eine vertraute stereotype Inhaltsfigur handelt, die im vorliegenden Kontext passend 
ist. Schon das zweite Segment wird gemeinsam produziert; und zwar kommt Frau Zimmermann der Sprecherin sogar zuvor $(4,10)$. Das dritte Segment - ein Kommentar - schließlich wird zu einem Gruppenereignis. Ingesamt fünf Sprecherinnen formulieren den Kommentar, mit einer weitgehenden Uberlappung der ersten Redebeiträge. Frau Wichmer und Frau Zimmermann antizipieren den Kommentar; sie starten fast gleichzeitig mit Frau Held, auf jeden Fall vor dem zentralen Formulierungsteil nimmer kumme. Frau Wichmer behält allerdings ihre Singsang-Intonation bei $(4,14)$ und erscheint damit nicht völlig in das kollektive formelhafte Sprechen integriert. In den Äußerungen der übrigen ist die Aussagemodalität der Selbstverständlichkeit durch die Formulierungsund Sprechweise deutlich markiert, und die kondensierenden Formulierungsverfahren werden in den Reformulierungen progressiv angewendet (vielleisch wird getilgt und garnimmer zu nimmer verkürzt).

Diese Sequenz stellt einen anderen Typ gemeisamen formelhaften Sprechens dar. Es handelt sich um 'Kommentieren im Chor' mit weitgehender inhaltlicher und ausdrucksseitiger Übereinstimmung der Äußerungen.

\subsection{Abschließende Bewertung}

Frau Zimmermann spricht das abschließende Urteil in der Bewertungsfrage, die von den Externen (Ingrid und Frau Kranz) und indirekt von Frau Wichmer mit ihrer Parteinahme aufgeworfen worden ist: $\operatorname{demm} w u=s$ : so" gemach werd demm gherd $=s$ ned onnerschd. Die Reaktionen darauf realisieren ein anderes Muster des gemeinsamen formelhaften Sprechens.

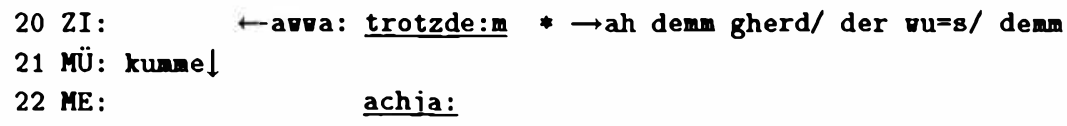

$23 \mathrm{ZI}$ : vu=s: so" gemach deam gherd=s ned onnerschd

$24 \mathrm{KU}$ :

25 WH:

e:ve:

26 MÜ: ah des is klarl ha ja\

$27 \mathrm{ZI}$ :

dem gherd=8 doch

ned annerschd

$28 \mathrm{KU}$ :

29 MÜ: loßd sisch des gfallel

do havee mer kä

30 IN : LACHT

S. 5

$1 \mathrm{ZI}:$ nei:n† nixl nix

$2 \mathrm{SU}$ :

aaa

$3 \mathrm{KU}$ : bedengge $\downarrow$

$\underline{\text { kä bedauern }}$

$4 \mathrm{KR}$ :

5 IN :

der läßd

un vie soll ma den etz sol 


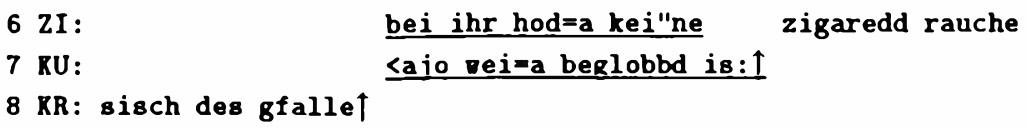

In dieser Sequenz variieren die Formulierungen maximal, und die Äußerungen unterscheiden sich auch funktional. Die Sequenz entspricht der Inszenierung eines Urteilsschemas mit verteilten Rollen:

- Frau Zimmermann formuliert eine allgemeine Sentenz, die nach dem Muster "wer ... der" gebaut ist $(4,20 / 23)$. Diese Sentenz drückt einen Aspekt der in der Filsbachwelt geltenden sozialen Kategorien für Eheverhältnisse aus: Ein "Hampelmann” ist nicht zu bedauern.

- Frau Kunz und Frau Wichmer reagieren mit zwei Varianten bedingungsloser Zustimmung (e:we: und ah des is klarl; 4,24/25), welche die Gültigkeit und Anwendbarkeit des allgemeinen Satzes bestätigen.

- Frau Müller formuliert die singuläre Prämisse (loßd sisch des gfalle $\downarrow ; 4,29$.

- In Überlappung damit formuliert Frau Zimmermann die Konklusion (4,27; vgl. auch den Formulierungsansatz in 4,20/23), indem sie den Folgesatz der allgemeinen Formel mit einer Markierung der Relevanzhochstufung doch reformuliert. Frau Zimmermann zeigt an, daB die SchluBfolgerung zu ziehen ist.

- Frau Kunz formuliert die praktische Konsequenz und demonstriert die Bewertungssicherheit des apodiktischen Urteils $(4,28 / 5,3)$.

- Diese Formel wird von Frau Zimmermann und Frau Schumann mit zwei Varianten bedingungloser Zustimmung unterstützt: nein $\uparrow$ nix $\downarrow$ und aaa $(5,1 / 2)$.

\subsection{Zusätze, Belege, Problematisierungsversuche}

In der Folge erscheinen keine Sequenzen gemeinsamen formelhaften Sprechens mehr, dafür aber eine Reihe von weiteren Thematisierungen und Problematisierungsversuchen, bei denen die Präferenz der Filsbachfrauen für formelhaftes Sprechen wirksam bleibt und dazu führt, daB Problematisierungsversuche nur bedingt Erfolg haben und da $B$ immer wieder in die Interaktionsmodalität der selbstverständlichen Sicherheit zurückgeleitet wird. Hierbei kommen u.a Sequenzen zustande, in denen ernsthafte, nicht-formelhafte, auf Detaillierung und Differenzierung gerichtete Äußerungen mit formelhaften beantwortet werden.

\section{(a) Weitere Belege}

Anschließend an die Bewertungssequenz versucht Frau Kranz, explizit Ottos Abweichung vom Normalbild eines Mannes zu thematisieren: der läßd sich des gfalle $\uparrow(5,4 / 8)$. Dieses Thema hat zunächst kaum Erfolg. Außer einer kurzen stereotypen Äußerung von Frau Kunz (ajo wei=a beglobbd is: $\uparrow ; 5,7$ ) erfolgt keine Reaktion. Dieses Thema wird erst durch Insistieren der Externen später durchgesetzt. Zunächst bringen Frau Zimmermann und Frau Held weitere Be- 
lege für Ottos Unterdrückung vor. Zwei der in der Sequenz der formelhaften Charakterisierungen verwendete Teilthemen erscheinen hier wieder: Zigaretten und Essensentzug. Diese Themen werden in formelnaher Weise formuliert:

S. 5

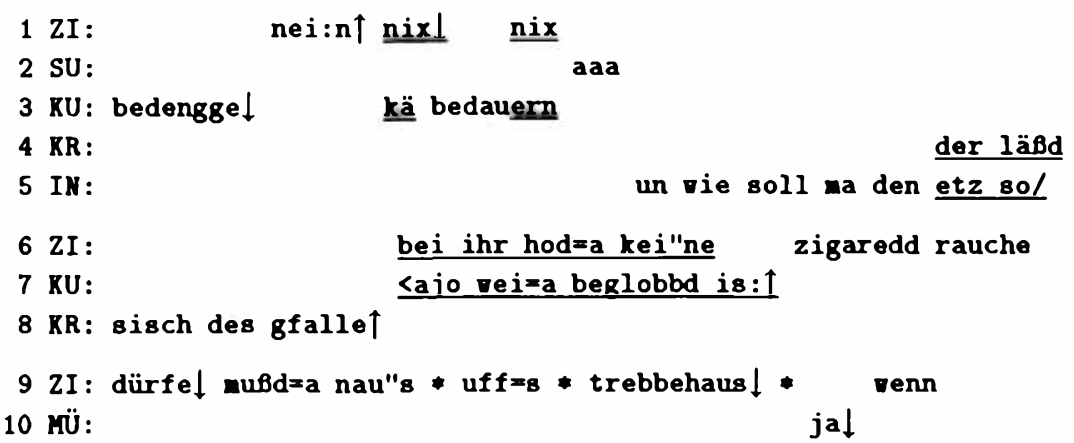

$11 \mathrm{ZI}$ : avva bsu"ch do var $\downarrow$ die" havve raache

12 MÜ: oooh

$13 \mathrm{ZI}$ : kenne LACHT

$14 \mathrm{HE}$ : un un der ihr mudda vo se noch

15 WH: LACHT do hädd=isch arva vas gsacht |

16 HE: gelebd hod $\uparrow$ die var noch

17 HH: die var genauso niserabel gell $\uparrow$

18 HE: schlima: $\uparrow$ un die hod vun ihra/ vun dema soim

19 MÜ: noch schlima var noch/

20 HE: geld gelebd venn er ovends noch vas esse gevolld hod hod=a

21 HE: ni"schds mehr griggd $\downarrow$ om on fernseh:

22 MÜ: ja jal

$23 \mathrm{KR}: \quad$ ja vas is=n des

24 LM: $\quad \underline{\text { ah ja }}$

$25 \mathrm{KR}:$ für=n mann $\uparrow(\ldots \ldots \ldots \ldots)$

26 IN : Das iszen des fürzn mann $\uparrow$ frag ich mich

Beide Themen werden in Form von generalisierten Geschichtenkondensaten behandelt. Die Formelnähe zeigt sich in der Anwendung bestimmter Verfahren der Kondensierung und Konturierung. Bei der Thematisierung der Zigaretten wird das antithetische Muster angewendet, wobei beide Teile durch eine zweiteilige Formulierung realisiert werden:

bei ihr hod=a kei"ne zigaredd rauche dürfe $\downarrow$

mußd=a * nau"s uff=s * trebbehaus $\downarrow$ * 
venn avga bsu"ch do var $\downarrow$

die" havge raache kenne LACHT $(5,6-5,13)$

Bei der Thematisierung des Essens erscheint wieder das Muster der wenndann-Konstruktion, eingebettet in eine übergeordnete konzessive Konstruktion („obwohl sie ..."):

un die hod vun ihra/ vun den soin geld gelebd

venn er ovends noch vas esse gevolld hod

hod=a ni"schds nehr griggd $\downarrow$

on on fernseh: $(5,18-5,21)$.

Die Aussparung der konzessiven Konjunktion ist ein Schritt der Kondensierung. Die Expansion mit der genaueren Situationsbestimmung om fernseh: als Explizierung zu owends geht der vorher schon erreichten Kondensierung jedoch entgegen.

(b) Nachfragen zu Otto

Die wiederholten, aber gar nicht oder nicht informativ behandelten Fragen von Frau Kranz und Ingrid nach der besonderen Verfassung von Otto werden jetzt sehr nachdrücklich gestellt und lösen eine weitere Charakterisierungssequenz aus. In ihrem Verlauf zeigt sich eine Divergenz in der Interaktionsmodalität zwischen den Fragestellerinnen und den Gruppenmitgliedern: Die Gruppenmitglieder bleiben auf dem Niveau der formelhaften oder formelnahen Charakterisierung:

S. 5

$21 \mathrm{HE}: \mathrm{ni}$ "schds mehr griggd $\downarrow$ gü:

22 no: ja ja $\downarrow$

$23 \mathrm{KR}$ :

24 LM:

ah ia

ja vas is=n des

$25 \mathrm{KR}: \mathrm{für}=\mathrm{n} \operatorname{mann} \uparrow(\ldots \ldots \ldots \ldots)$

26 IN : Das is=en des für=n mann $\uparrow$ frag ich mich

$27 \mathrm{ZI}: \quad \geq(\ldots \ldots \ldots \ldots \ldots)$

28 HE: des: is=n debb hear $\uparrow$

$29 \mathrm{KU}:$ ah ia des: en de"bb

30 HH: $\quad$ der hod/ der/ der hod vun Vietnam ä

31 IN : auch |

S. 6

1 แH: bissel vas ghabd ääh-

2 ME:

>sie ho=do sovas

3 IN :

ach des is=n solda: $\mathrm{d} \uparrow$ 


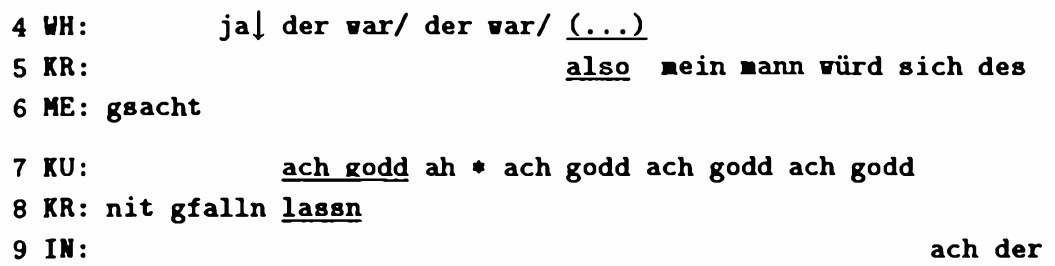

$10 \mathrm{ZI}$ :

ahja

$11 \mathrm{KU}:$

der

$12 \mathrm{HH}$ :

ahja der hod vas $\downarrow$

13 IN : is virklich ä bissel bscheu/ also-

$14 \mathrm{KU}$ : hod=n schdisch ins griene $\downarrow$

15 HH :

avva ven=ma nid=m schbrischd

16 IN :

ach 801

Die erste gemeinsame und nachdrückliche Initiative von Frau Kranz und Ingrid löst eine Folge von Antworten aus, die auf der Ebene der oben schon von Frau Kunz gegebenen Antwort <ajo wei=a beglobbd is: $\uparrow(5,7)$ liegen. Es sind kurze formelhafte Charakterisierungen mit der Aussagemodalität der Sicherheit:

- des: is $=n$ debb $(5,28)$

- ah ja des=s en de"bb $(5,29)$

Eine erste andeutende Hintergrundinformation der hod vun Vietnam ä bissl was ghabd veranlaßt Ingrid zu einer Nachfrage, die aber an dieser Stelle nicht beantwortet wird, sondern erst viel später durch Frau Zimmermann:

der var soldad $\downarrow$ *

un var in Korea * nit $\uparrow$ der"

un veil er dreiBisch jahr bei:n *

vie seschd ma mir sache * beim bund beim milidär var *

is er jo donn endlasse vorre nit $\uparrow(7,15-7,19)$.

An der vorliegenden Stelle kommen nur andeutende, ausweichende und unsichere Auskünfte. Die spätere Auskunft von Frau Zimmermann, daß Otto im Koreakrieg war, läßt rückwirkend auch die Formulierung der hod vun Vietnam $\ddot{a}$ bissl was ghabd $(5,30 / 6,1)$ in klarerem Licht erscheinen. Die Ungenauigkeit der Ortsangabe geht darauf zurück, daß Otto für die meisten Gruppenmitglieder gar nicht so wichtig ist, sondern daß er vor allem als Partner von Gerda interessiert. Deswegen können die Gruppenmitglieder sich auch mit der ungenauen Information zufriedengeben: Vietnam ist eine Metapher für „Krieg der Amerikaner". Insgesamt zeigt diese Bearbeitung der Fragen von Ingrid und Frau Kranz, daß die Gruppenmitglieder die Veränderung der Interaktionsmodalität zur ernsthaften Klärung der Fakten nicht ratifizieren.

Eine zweite Initiative in dieser Richtung von Frau Kranz wird von Frau Kunz mit einer Formel der „reinen Bewertung” beantwortet, d.h. ohne jede Zusatz- 
information: ach godd ah * ach godd ach godd ach godd $\downarrow$ (6,7). Diese Äußerung manifestiert noch einmal die in allen voraufgehenden Kommentaren zu Otto enthaltene Bewertung, die auch früher schon durch ohje ohje $(1,10)$ oder ach gott $(2,21)$ ausgedrückt wurde.

Die Folgeinitiative von Ingrid verschiebt erneut die Interaktionsmodalität in Richtung auf die Klärung der Fakten wirklich; $(6,13)$ sie hinterfragt damit die Charakterisierungsformeln vom Typ des is $=n$ debb. Die beiden Antworten sind formelhaft und geben keine konkreten Auskünfte:

- ahja der hod was $(6,12 / 14)$

- der hod=n schdisch ins grienel $(6,21)$.

Diese Äußerungen sind als Formeln für deviantes Verhalten zu verstehen. In dieser Funktion verwendet Frau Kunz die Formel vom schdisch ins griene häufiger, z.B. später im Gespräch auch für Gerda: des siehd doch än normaler [...] daß die än schdisch ins griene hod. In dieser Passage wiederholt sich mehrfach die Beantwortung einer ernst und faktenorientiert gemeinten Initiative mit einer formelhaften, auf einfache Typisierung in der Modalität fragloser Sicherheit orientierten Reaktion. Diese Abfolge war bereits am Ausgangspunkt des Thematisierungsbogens erschienen, als Ingrid nach Otto fragt: der mann $\uparrow * \operatorname{der}$ ehemann $\uparrow$. Dahinter steht eine Präferenz der Gruppenmitglieder für formelhaftes Sprechen bei der Formulierung von sicheren, einfachen Kategorisierungen im Tratsch. Diese Präferenz wird jetzt auffälliger, weil sie gegen die Initiativen der insistierenden Externen beibehalten wird. Die Versuche, das Thema "Otto" in differenzierterer Weise zu behandeln und den Bedarf an Hintergrundinformationen zu decken, stoßen immer wieder an eine Grenze. Hier wird im Ansatz etwas von dem Interaktionspotential der Ausgrenzung von Fremden deutlich, das mit formelhaftem Sprechen verbunden ist (vgl. dazu weiter unten Kap. 6.). Es sind immer nur einzelne Gruppenmitglieder, die in kooperativer Weise hin und wieder die Externen aufklären. In diesem Fall übernimmt Frau Zimmermann diese Rolle und liefert die oben schon zitierten Informationen über Ottos Vergangenheit als Soldat.

(c) Ein Versuch zu komplexerer Typisierung

Nicht nur Externe haben Schwierigkeiten, sich gegen das routinisierte Gesprächsmuster durchzusetzen. Frau Wichmer versucht eine Art Rehabilitierung Ottos. Sie plädiert dafür, Otto auch als selbstständiges Individuum zu sehen. Bei ihrer Initiative zu einer komplexeren Typisierung erscheinen deutliche Formulierungsmerkmale der Problematisierung. Aber dieser Ansatz wird abgeblockt.

S. 6

15 H:

avpa ven=ma mid=n schbrischd

16 IN :

ach sol 
17 ZI:

18 HH: ned $\downarrow$

$19 \mathrm{KR}$ :

oh der

20 IN :

$\underline{\text { nänäl }}$

$\underline{\text { nä/nä }}$

ja:

kennsch $\underline{d u=n \uparrow}$

$21 \mathrm{ZI}$ : schbrischd ava ned viel $\downarrow$

$22 \mathrm{KU}$ :

nää nää

$23 \mathrm{HH}:$

Sia: agva venn/ avva venn=a aus

$24 \mathrm{HE}$ :

ja

25 UH: sisch raus gehd * der schbrischd sehr nedd und äh-

$\begin{array}{ll}26 \mathrm{ZI}: \quad \text { ja: des schdinad } & \text { der is } \\ 27 \mathrm{KU}: & \end{array}$

28 rü: jaja घenn=a aus/

29 KR: ia

$30 \mathrm{ZI}$ : net $(\ldots)$

$31 \mathrm{HE}$ : ja ja ja

$32 \mathrm{HH}: \quad$ venn sie ned zuherd $\uparrow$

33 Mü : oh ja nä nä

$\mathbf{K}$ :

DURCHE INANDER

S. 7

1 WH: der/ venn sie ned zuherd $\downarrow$

$2 \mathrm{KR}$ :

ja! $* j a \downarrow$ vir ham

$\mathrm{K}$ :

MIKRORAUSCHEN

Die Äußerungen wenn er was redde derff $(6,27)$ und wenn sie ned zuherd $(6,32 / 7,1)$ ordnen Otto sofort wieder in das Abhängigkeitsverhältnis des Hampelmanns von seinem Hausdrachen ein. Diese Formulierungen stellen eine weitere Variante der Kontrolle Ottos durch Gerda dar; sie sind inhaltlich stereotyp und haben aufgrund der Kürze und der intonatorisch markierten Ausdrucksmodalität der Sicherheit Merkmale des formelhaften Sprechens. ${ }^{26}$

(d) Eine Wiedergutmachung

Am Ende des Thematisierungsbogens steht eine Formelreminiszenz, d.h. die andeutende Erinnerung an formelhaftes Sprechen, und zwar in diesem Fall an gemeinsames formelhaftes Sprechen. Der AnlaB dafür ist eine kleine Turbulenz im Gespräch. Frau Wichmer hat einen weiteren Versuch der Parteinahme für Otto realisiert, indem sie eine Geschichte zum Thema „Wie Otto die Gerda mit

26 Die kurz darauf folgende Äußerung gege den is nix zu sache $(7,6)$ von Frau Zimmermann, die generell die Position der Norm und der klaren Verurteilung von Otto vertritt, ist ein Zugeständnis in der spezifischen Interaktionssituation. Sie akzeptiert und honoriert damit die Bemühungen von Frau Kranz um ein klares Bild von den Verhältnissen in der Filsbachwelt. 
dem Geld mal reingelegt hat" anfängt: der hod se awwa mol gonz schä bschi"sse $(7,22)$. Die Erzählankündigung stöBt auf großes Interesse bei Frau Kranz, die ebenfalls in der Einschätzung von Otto vorsichtiger ist als die Gruppenmitglieder; die Erzählung wird aber von Frau Zimmermann abgeblockt mit dem Hinweis darauf, daB Otto sein Geld immer sofort abgeliefert habe und daß sie das selber verfolgt habe: $i$ "sch war schunn debei wo=a=s geld ghold hod un hot $=s=$ ere hiegeleggd $\downarrow(7,28 / 8,1)$. Frau Wichmer verliert den Kampf um das Erzählrecht. Der Vorfall ist undramatisch, aber nicht harmlos, weil zwischen Frau Wichmer und Frau Zimmermann zu dieser Zeit ein Konkurrenzkampf um die Dominanz in der Gruppe und um Fragen des sozialen Stils stattfindet. Nach der Unterbrechung wendet sich Frau Wichmer von Frau Zimmermann ab und anderen Beteiligten im Hintergrund zu und bewundert in betonter Weise deren Bastelarbeiten: oh des is schö:n\ $(8,15)$. Als sie sich nach einer ganzen Weile zum ersten Mal wieder als Sprecherin in der vorigen Gesprächskonstellation mit Frau Zimmermann zurückmeldet, spricht diese sie sofort an:

S. 9

22 ZI: <fra Wi"chmer vas verdd=n der zur silverhochzeit zu fresse

23 ZI: krigge $\uparrow$

LACHT

24 WH: hoffenl/ ah hoffenlisch kriggd=a ä rumgchdek

25 WH: un kä/ kä * un kää * eier mid schingge $\downarrow$

26 IN :

LACHT die katz odda

$27 \mathrm{ZI}$ :

*de Oddo*

die hod/

nä

$\mathrm{K}$ :

*LACHEND*

28 WH:

de Oddo $\downarrow$

29 IN : de Oddo $\downarrow$

ach sol* die havge kä katz $\uparrow$

Stereotype Inhaltsfiguren und Formeln sind als Gesprächsobjekte ständig verfügbar; sie gehören zum gesicherten Bestand einer Sprechergemeinschaft und gestatten eine Beteiligung ohne besondere Anstrengungen oder Risiken. Sie sind insofern geeignet für bestimmte Aktivitäten wie thematische Initiativen nach Flauten oder Veränderungen der Beteiligtenkonstellation. Frau Zimmermann bietet Frau Wichmer mit ihrer Frage nach dem Essen, die manifest an die voraufgehende gemeinsame Behandlung des Themas anknüpft, die Chance zu einer aufwandsarmen und völlig risikofreien Beteiligung. Das ist eine manifest kooperative Aktivität, die zudem Bezug nimmt auf die frühere, von wechselseitiger Bestätigung bestimmte Gesprächssituation und damit eine Fortsetzung der spannungsfreien Beziehung anbietet. Frau Zimmermann initiiert eine Beziehungsreparatur. Frau Wichmer ratifiziert die Reparatur durch ihre korrespondierende, die Kernformel der voraufgehenden Themenbehandlung reproduzierende Reaktion.

Hier deutet sich im übrigen ein weiterer Typ von gemeinsamem formelhaften Sprechen an: Frau Zimmermann formuliert mit ihrer Frage eine Einladung zum 
Phantasiespiel; bei einer solchen Thematisierung malen sich die Beteiligten eine typische Szene mit lustigen Übertreibungen aus (vgl. Kap. 5). An dieser Stelle kommt aber keine weitere Behandlung des Themas in Gang; auch die beiden Hauptakteure machen keine Fortsetzungsversuche. Das ist ein Indiz dafür, daB für beide die Funktion der Beziehungsreparatur im Vordergrund steht.

\subsection{Zusammenfassung}

Die Ergebnisse der Gesprächsanalyse sollen hier kurz zusammengefaßt werden:

(a) Die Analyse hat gezeigt, wie das Gespräch durchsetzt ist mit formelhaften und formelnahen ÄuBerungen. Dabei gibt es zwei Pole für die Deutlichkeit und Erkennbarkeit der Formelhaftigkeit. Auf der einen Seite stehen Formulierungen,

- die klar vom Kontext als selbständige Äußerungen abgetrennt sind,

- die bestimmten Formaten entsprechen, hier insbesondere Charakterisierungen als Kurzkommentare, und die zusätzlich durch typische ProsodieMuster gekennzeichnet sind,

- die in Folgen formelhafter Äußerungen erscheinen, bei denen u.U. die Formelhaftigkeit als Formulierungsweise interaktiv etabliert wird; dies geschieht durch die progressive Anwendung der Formulierungsverfahren für formelhaftes Sprechen und durch klare Korrespondenz von formelhaften Folgeäußerungen.

Auf der anderen Seite stehen Formulierungen, die textuell integriert sind und bei denen die Formulierungsverfahren für Formelhaftigkeit nur partiell angewendet sind, die aber immer noch als formelnah erkennbar sind (u.a. wegen bestimmter Konstruktionsmuster wie der antithetischen Formulierung oder wenndann-Konstruktionen).

(b) Es gibt eine Reihe von Sequenzmustern für formelhaftes Sprechen. Im Beispieltext sind folgende Formen für gemeinsames formelhaftes Sprechen aufgefallen:

- die Runde funktional paralleler, einem Thema zugeordneter Kommentare, d.h. ohne thematische Progression, aber mit rhematischer Ausgestaltung (Auflistung von Eigenschaften eines Typs); das kann, wie im Beispielfall, verbunden sein mit Elementen eines chorischen Zustimmens/Kommentierens;

- die Runde funktional paralleler, übereinstimmend formulierter Äußerungen ohne thematische Progression und ohne rhematische Ausgestaltung (Kommentieren im Chor);

- die Inszenierung eines kleinen Handlungsschemas, z.B. eines Urteilschemas, durch eine Reihe von funktional differenzierten Äußerungen mit thematischer Progression. 
Gegenstand dieser formelhaften Sequenzen sind einfache Kategorisierungen und Bewertungen in einer Interaktionsmodalität der fraglosen Sicherheit.

Daneben gibt es Formen von Sequenzen, in denen nicht alle Beteiligten formelhaft sprechen, sondern in dieser Hinsicht eine Asymmetrie besteht. Auffällig ist das, wenn formelhafte Äußerungen der genannten Art auf nicht formelhafte, auf Faktizität orientierte antworten. Sowohl das gemeinsame als auch das asymmetrische formelhafte Sprechen verdeutlicht die starke Präferenz der Filsbachpopulation für formelhaftes Sprechen bei einfachen Kategorisierungen im Tratsch.

Bei der Analyse haben sich zwei Hinweise auf wichtige Eigenschaften des formelhaften Sprechens ergeben, die im Beispiel nicht deutlich repräsentiert waren und die deshalb noch anhand zusätzlicher Materialien dargestellt werden sollen:

- wie sich die Realitätsbindung und Konkretheit des formelhaften Sprechens zur Freiheit des Spiels verhält, die bestimmte Kommunikationsformen kennzeichnet (vgl. 5.);

- welche Merkmale formelhaftes Sprechen in abgrenzender Funktion hat (vgl. 6 .).

\section{Spiel und Witz}

Wie dargestellt wurde, haben einige der oben analysierten Sequenzen formelhaften Sprechens schon Züge der spielerischen Ausgestaltung eines Stereotyps, bei der nicht nur das gesicherte Typisierungswissen präsentiert und die Geteiltheit noch einmal bestätigt wird, sondern bei der auch der Reichtum an Formeln sowie deren Treffsicherheit und Witz unter Beweis gestellt werden. Beim Übergang zur spielerischen Ausgestaltung gibt es offensichtlich zwei Stufen. Zum einen werden Listen von Eigenschaften zusammengestellt, wobei die verwendeten Formeln bereits geprägt und routinisiert sind, zum anderen werden in einem Phantasiespiel vorgestellte Situationen zur Charakterisierung benutzt. Zur ersten Gruppe gehört das folgende Beispiel, in dem über Gerda gesprochen wird:

10 IN : is die so groB $\uparrow$

$11 \mathrm{HE}$ : aaah noch emol so grob vie isch *

$12 \mathrm{kU}:$

$13 \mathrm{HH}$ : $(\ldots)$ die is

$14 \mathrm{SU}:$ (die müßtn se sehn?)

$15 \mathrm{KU}:$ gro:"B *

16 WH:

un so e paar fieb * zvee" händ vun

$17 \mathrm{KU}$ : die brauchd=n vaffeschoi fer ihr händ

18 HH: moine (LACHT)

(LACHT) 
$19 \mathrm{KU}$ : $\rightarrow$ venn die ään änni noiballerd demm verd=s

20 UH: än vaffeschoi (LACHT)

21 IN :

LACHT

$22 \mathrm{KU}:$ nachd $\downarrow$

23 แH:

24 MÜ:

die hod nämisch e paar kohleschaufle LACHT

25 Wh: un fie"B her $\uparrow$ (LACHT)

$(2036 / 36)$

Diese Art der bildhaften grotesken Charakterisierung in spielerischen Runden führt zu immer reicheren Listen von Eigenschaftsformeln. Ein Jahr später wird z.B. bei einer solchen Gelegenheit Gerda so dargestellt:

- pfode ... die brauchd=n waffeschoi [...]

- fieß * die konn iwwer de Neggaa laafe

- vun derre meschd isch känni gschosse krigge * do geh isch dreimal riggwärts raus

- ̈̈ großi gosch un nix dehinner,

Und von Otto wird gesagt:

- $\operatorname{der} m i=m$ schdecksch $u$

- der hod ä maddscheib

- der hod=n schuß ins griene.

Hier wird ein im sozialen Milieu der beobachteten Gruppe gebräuchliches, aber in seiner Prägung nicht geschichten-gebundenes Formelrepertoire in die Charakterisierungsspiele integriert.

Einen Fall der zweiten Art, d.h. der Ausgestaltung einer Situation, stellt das folgende Beispiel dar. Auch hier ist zu beobachten, wie die Charakterisierung im Tratsch in ein Phantasiespiel mündet:

8 rü: die sachd zu dem Oddol geh ä schdund nunner in de keller $\uparrow$ un

9 rü: in ere schdunn konnsch vidder ruffkume- in der zeid hot $s=e n$

10 IN : vas macht der ne

$11 \mathrm{SU}:$

12 MÜ: onnere $\downarrow$

$13 \mathrm{KL}$ : LACHT $\leftarrow$ das gibt es $\uparrow$

14 IN : schdund da unde $\uparrow$

$15 \mathrm{SU}$ :

16 MÜ:

ah isch vääß=s net

$\mathbf{R}:$

der hat e anner da untn

\section{LACHEN}


$17 \mathrm{SU}:$ ah der is doch da"bbisch

$18 \mathrm{HI}$ : der laufd immer so": hie

$R$ : STIRIFEGEUIRR

19 IN :

(..)

$20 \mathrm{SU}$ : is die schunn ferdisch $\downarrow$

$21 \mathrm{HI}$ : un bis der unne in keller is $\uparrow$

22 HI: is e halvi schdundl un bis er vidder ovge

$\mathrm{R}$ : STARKES LACHEN

23 2I:

24 HI : noch emol e halvi schdund $\downarrow$

$(2036 / 62 a)$

Die wesentlichen Elemente der Gesprächsentwicklung zum Phantasiespiel sind hier:

(a) Am Beginn steht eine generalisierte Szenendarstellung von Frau Müller (8/9), die bereits eine Überspitzung in der Art von "gut erfundenen Details" enthält ("Otto eine ganze Stunde in den Keller schicken").

(b) Die Frage von Ingrid (10/14) löst die Steigerung zum Phantasiespiel aus; auf die Frage werden eine Reihe unterschiedlicher Antworten gegeben.

(c) Eine Antwort von Frau Müller (16): Sie muB „passen”.

(d) Eine Antwort von Frau Schumann (15): Sie formuliert eine Umkehrung der Situation entsprechend dem Topos vom "siegreichen Verlierer” bzw. vom "Triumph des Opfers"; diese Formulierung entfernt sich sehr weit vom Wissen über die Situation und dem festen Bild von Otto; der Erfolg bei den anderen Teilnehmerinnen ist unsicher (das Stimmengewirr ist nicht zu deuten).

(e) Eine Antwort von Frau Hild (18): Sie knüpft an konkretes Wissen an (Ottos langsamer Gang: er wird nonverbal imitierend als extrem langsam verdeutlicht) und formuliert eine weitere Ubertreibung (Otto braucht sich nicht im Keller aufzuhalten, sein Weg dauert lang genug).

(f) Frau Schumann reagiert zunächst auf die Eröffnung von Frau Hild mit einem Standardkommentar (17), der ganz unspezifisch und nicht in das Phantasiespiel integrierbar ist, liefert dann (20) eine gelungene Fortsetzung der begonnenen zweiteilig konstruierten Äußerung von Frau Hild (19); die Fortsetzung enthält eine weitere Übertreibung und zugleich ein weiteres inhaltliches Element (Gerda als „schnelle Frau”), das mit dem Gerda-Bild kompatibel ist; diese Antizipation hat großen Erfolg und übertrifft die von Frau Hild formulierte Pointe.

Der formelhafte Tratsch mündet immer wieder in routinehafte, spielerische Sequenzen der geschilderten Art. Bei den Phantasiespielen im Rahmen des Tratsches ist zu beobachten, daß auch sie noch sehr direkt an den Erfahrungs- und 
Geschichtenhintergrund gebunden bleiben. Eine ihrer Erfolgsbedingungen ist, $\mathrm{da} B$ sie treffsicher sind in bezug auf die Eigenschaften der besprochenen Personen und die Lebensbedingungen in der sozialen Umwelt. Diese Art des spielerischen Austausches steht in dieser Hinsicht in Kontrast zu phantasiereichen, nicht mehr realitätsgebundenen Wortspielen oder zu Witzen.

Witze sind für die beobachtete Gruppe eine attraktive Textgattung, vor allem sexuelle Witze, und sie haben im ProzeB der Gruppenkonstitution eine erhebliche Rolle gespielt. Sie sind als vorformulierte Texte jederzeit als Unterhaltungsmittel verfügbar, und zwar ohne eine weitergehende Kenntnis der spezifischen Lebenswelten und Biographien der Beteiligten. Zugleich beinhalten sie aber viele Elemente, die zur Bestimmung des sozialen Stiles relevant sind wie Normen und Tabus, Situationsmodelle und Kategorien des Komischen usw. Witzeerzählen eignet sich insofern auch zur Aushandlung von Eigenschaften des sozialen Stils, und es ist von der Gruppe auch ausgiebig dazu benutzt worden. Aber das Erzählen von Witzen symbolisiert die Relevanzen der eigenen sozialen Welt auf eine sehr gebrochene Weise und mit Texten, für die konstitutiv ist, daß sie fremdformuliert sind (vgl. auch Beitrag 9 in diesem Band). Von daher ist auch ganz natürlich, daB die Gruppenmitglieder die Interaktionsmuster Witzeerzählen und Tratschen nicht vermischen, auch nicht, wenn der Tratsch spielerische Züge annimmt. Das gilt jedenfalls für die Kernmitglieder, während Hinzugekommene wie Frau Schumann in dieser Hinsicht wiederholt Schwierigkeiten haben. Die Textmuster der in der Gruppe präferierten Witze und die darin enthaltenen Kategorien des Komischen sind wesentlich allgemeinere Wissensbestände als das spezifische Weltwissen, das im Tratsch vorausgesetzt wird. Der Erwerb der wichtigsten Witzschemata und das Akzeptieren der darüber symbolisierten Eigenschaften des sozialen Stils sind eine wesentliche Stufe der Gruppenintegration, die Frau Schumann z.B. relativ schnell erreicht hat. Sie stammt aus Mitteldeutschland, und ihr fehlen historische und kulturelle Hintergründe, aber sie hat eine anerkannte Kompetenz für Witze und witzige Bemerkungen und teilt die in der lokalen Kultur geltenden Kriterien des Komischen. Das formelhafte Sprechen im Tratsch dagegen, und hier speziell die Verwendung von selbstgeprägten, geschichtengebundenen Charakterisierungsformeln ist für Hinzugekommene eine schwierige Klippe.

Im folgenden Beispiel beteiligt sich Frau Schumann am Tratsch über Otto, den die anderen wesentlich mit Charakterisierungsformeln und eng verwandten Formulierungen bestreiten, mit einer Witzreminiszenz (der $m u ß ; 3,6)$. Diese ist durch steigende Intonation und Emphase hervorgehoben, wird von den anderen jedoch nicht gewürdigt; es wird keine Fortsetzung im Sinne der Witzbehandlung oder des kollektiven formelhaften Sprechens ausgelöst. Frau Schumann insistiert $(3,3)$, liefert schlieBlich unaufgefordert den Witz nach und versucht angesichts eines relativ bescheidenen Lacherfolges, die für Witzeerzählen typische Honorierung auch explizit zu bekommen (der=s gu:d gell $; ; 4,2)$. Es kooperiert aber nur die anwesende Externe, Ingrid (der $=s$ gud; 4,4), während die 
übrigen das Ereignis weitgehend übergehen und inhaltlich wie der Thematisierungsweise nach den Tratsch fortsetzen (MÜ: die hod als onnere do; 3,24).

S. 3

$6 \mathrm{SU}:$

der mu"B: $\uparrow$

7 MÜ:

vas nääne se vie dere

8 HI : daB der ait der zusameleve kann $\uparrow$

9 rü: Gerda ihr mudder noch gelebd hod gas do los var

$10 \mathrm{ZI}$ :

der is verhei"rad aid ere

11 MÜ: ou: : liever

12 ZI : un der därf/ <bei den därfe se nix sa:che $\uparrow$ nei:n $\uparrow$

13 SU: der nu:B $\uparrow$

$14 \mathrm{ZI}$ : des is seine fraul

$15 \mathrm{SU}:$

16 Mü: godd der guggd in die vie=n=en schbiggel

17 2I : lie: ver godd

18 SU: vie der vitz $\uparrow$ veil ich eben sach der mu"B äh * konnt einer

19 IN : LACHT

$20 \mathrm{SU}$ : frühzeitisch hein $\uparrow$ von der arbeit $\uparrow$ überrascht seine frau nit sein $\mathrm{K}$ : DURCHEINANDER

21 SU: bestn freund in bett $\downarrow$ un da guckt=a sacht=a also: * $i$ "ch muß ja $22 \mathrm{ZI}$ :

23 SU: avga du:" $\uparrow$

24 Mü:

$\mathrm{K}: \quad$ LACHEM do schdehd=s doch uff

S. 4

$1 \mathrm{ZI}:(\ldots \ldots \ldots)$

2 SU: der=s gu:d gell $\uparrow$

3 rü: un do sach der Oddo nix die hod als onnere do

4 IN: der=s gud

$5 \mathrm{ZI}: \quad$ vas $\uparrow$

6 rü: dab die onnere hod $\downarrow$ * onnere ghabd hod die Ge"rda *

$7 \mathrm{HI}: \mathrm{ja}$

$8 \mathrm{ZI}$ : nönöl sachd (...)

9 rü: do sachd der Oddo nix der guggd in die noi vie=n=en

10 IN : vas die hot/ 
11 ZI: $\rightarrow$ nänää

12 MÜ: schbiggel

13 IN :

die hat annere männer ghabt $\uparrow$

$(2036 / 62 a)$

Das Besondere am Mißerfolg von Frau Schumann ist, daß den übrigen die Formulierung der muß durchaus als Formel vertraut ist, aber als eine geschichtengebundene, im Tratsch eingeführte Formel. Drei Jahre früher, noch ohne die Beteiligung von Frau Schumann, wird die Formel von den Gruppenmitgliedern beim Kommentieren im Chor verwendet:

$1 \mathrm{ZI}$ :

vas die dem sescht $\uparrow$

2 ZI: des mach=der $\downarrow$ nit $\uparrow$

$3 \mathrm{KU}$ :

ajo der mach=des des is än

$4 \mathrm{ZI}: \quad$ net $\uparrow$

der muB *ja ja *un/ *un do

$5 \mathrm{KU}$ : rischdischer (...)

$6 \mathrm{WH}: \quad \operatorname{der} \operatorname{muB} \uparrow$

(2035/05)

In diesem Beispiel entspricht der muß hinsichtlich der Kürze, des Kommentarformats und der intonatorisch markierten Aussagemodalität der Sicherheit den formelhaften Charakterisierungen. Der Mißerfolg von Frau Schumann beruht offensichtlich auf einer andersartigen Kontextualisierung, die durch die prosodische Hervorhebung ( $\operatorname{der} m u$ " $\beta \uparrow$ ) für die übrigen erkennbar wird.

\section{Soziale Abgrenzung durch formelhaftes Sprechen}

In den bisherigen Analysen ist formelhaftes Sprechen vor allem als Form demonstrativer Gemeinschaft, d.h. als Mittel der Symbolisierung der eigenen Welt und der sozialen Zusammengehörigkeit deutlich geworden. An einigen Stellen deutete sich dabei schon an, daB formelhaftes Sprechen in seiner sozialen Spezifik auch sozial ausgrenzend wirken kann. Nun gibt es auch Arten des formelhaften Sprechens, die gerade auf soziale Ausgrenzung oder auf Zurückweisung von Übergriffen anderer zielen. Dazu werden in der Filsbachwelt häufig lokal spezifische Sprüche verwendet. Diese sind - wie das bislang analysierte formelhafte Sprechen insgesamt - mit der Verwendung der lokalen Sprachlage verbunden. Bei ausgrenzender Verwendung von Formeln gegenüber Externen spielt diese Sprachlage häufig eine symbolisierende Rolle. Das ist auch bei dem folgenden Beispiel der Fall. Das macht die Analyse etwas komplexer, gibt zugleich aber auch Gelegenheit, die Verbindung zwischen den verschiedenen Symbolisierungsverfahren explizit herzustellen.

Ein Formeltyp, der speziell für die soziale Abgrenzung verwendet wird, sind Unsinnssprüche. Das folgende Beispiel „Handheb fer=n mehlsack” (handheb 
$=$ Henkel, Griff) enthält eine Erzählung über die Verwendung eines solchen Unsinnsspruchs.

Die Gesprächssituation ist dieselbe wie im Beispiel „Geschlossene Gesellschaft”, und es geht auch hier um die Auseinandersetzung mit Frau Hansen, der Leiterin einer Altentagesstätte (vgl. den voraufgehenden Beitrag). Die Erzählung ist Teil einer Kette von Thematisierungen dieses Konflikts; die Erzählung „Geschlossene Gesellschaft” liegt kurz vor der jetzigen Gesprächsstelle (vgl. den Textanhang zu Beitrag 3).

S. 19

1 ZI: härn se mol vas/ äh * jetz erzähl isch ihne mol neilisch *

2 ZI: sitze mir dordd auch nonda:chs un schdrigge die frau *

3 BA: $\quad$ Meyer

$4 \mathrm{ZI}$ : Meyer un isch * ner schdrigge dordd * jaja monda:chs

$5 \mathrm{KR}$ : bei: dort bei de

6 ZI: also unna de voch * sim=ma immer hie * un äh* do

$7 \mathrm{ME}:$ in dä"re roch

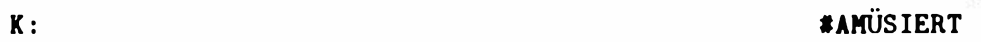

$8 \mathrm{ZI}$ : kund sie un sacht * \#as strücke se dönn- * no sach=die fra $\mathrm{K}$ : *GESPITZTER RUND *

$9 \mathrm{ME}:$ nas/

$10 \mathrm{ZI}:$ Meyer * hondheb fer e nehlsack

11 BA:

ah jo des=aam Monnemer

K : LACHEN

12 ZI: *des=8 gibd/ des gibd es*

K : $\quad$ *HOCH

13 BA: ausdrugg

$14 \mathrm{ME}$ : $\quad$ des hedde sie a gsagd

$15 \mathrm{ZI}$ : jetz hod se des ned verschdonne * nid $\uparrow$

16 BA: Monnemer ausdrugs

17 ME: des=n ausdrugg

18 IN: e handheb e handhebe

19 2I: e handheb fer=n/ se hondheib

20 ME: $\quad$ des gib=s doch ned des geeb ma

21 IN: für=n mehlsack *

S. 20

1 ZI: hot se gsachd e hondheib fer=n mehlsack nid $\uparrow$ un donn

2 2I: LACHT do is die hinnere un hod bei denne onnere

3 ME: vonn jemond so nei/ neigierisch is 
4 ZI: gfrogt vas des is

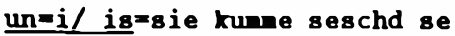

5 BA: e hondheb gellt

$6 \mathrm{ZI}$ : tah sie habn misch ja auf dn arn genomen*

$R$ : *STANDARD

7 ZI: Hir havge õ:gfonge zu lache ned $\uparrow$ $\mathrm{R}$ : LACHEND *

8 BA:

K: GELÄCHTER

un i"sch

ah schbass nub aa

$9 \mathrm{ZI}$ : hab donn gsacht moin bruder hedd gsach * en riggl fer=n

$10 \mathrm{BA}$ : 01 soi nid $\uparrow *$

11 ZI: gänsaasch $\mathbf{K}:$

taa:h*

12 BA: *HOCH*

13 IN:

vas denn $\uparrow$

14 ZI : LACHT

*des is vomoin äldschde bruder

$\mathrm{K}$ : *HOHE STIMAE

15 ME:

ah do:ch horsch emol/

16 LM: $(\ldots \ldots)$

17 ZI : $\Rightarrow$ des hod er gsacht* * gäns/ * vas gib=n vo=ma gsachd hod K: HOHE STIMM:

$18 \mathrm{ME}:(\ldots \ldots \ldots \ldots \ldots \ldots \ldots$

$19 \mathrm{ZI}$ : gas_gib=n des $\uparrow$ än riggel fer-n gänsaasch

$20 \mathrm{ME}:(\ldots \ldots)$

21 IN : du muschd/ * mir

Gliederung der Erzählung:

- 19,1: Erzählankündigung;

- 19,2-6: Situationshintergrund (Stricken in der Tagesstätte), mit kurzer Behandlung des Themas „Benutzung des problematischen Schauplatzes” ( $u n$ ner de woch sim=ma imma hie);

- 19,6-20,1: erste Konfrontation (neugierige Frage und zurückweisende Antwort), wobei das Nicht-Verstehen von Frau Hansen (für die der Unsinnsspruch ein Rätsel darstellt) hervorgehoben wird;

- 19,11-20,5: Erklärung des Spruchs für Ingrid;

- 20,1-20,7: Auflösung des Rätsels und zweite Konfrontation (Frau Hansen läßt sich von anderen das Rätsel lösen und stellt die Urheberin zur Rede);

- 20,7-20,12: Erklärung (Frau Zimmermann verdeutlicht den Formeltyp durch eine Variante);

- 20,13-20,21: Erklärender Kommentar in aktualer Gesprächssituation (ausgelöst durch Nachfrage von Ingrid). 
Es handelt sich um eine Geschichte von „uns und den anderen”, die in einer Situation "in unserer eigenen Welt" erzählt wird, und in entsprechender Weise wie vorher bei der Erzählung "Geschlossene Gesellschaft" ist die dargestellte soziale Konstellation in der Gesprächssituation präsent (Handlungsträger sind Kernmitglieder der Gruppe, und eine - akzeptierte - Institutionenvertreterin ist anwesend).

Die Distanz zwischen „uns" und den „anderen” wird dabei an entscheidenden Punkten der Erzählung durch symbolisierende Variation sehr klar verdeutlicht: Die Erzählerin gibt zunächst die Rede von Frau Hansen und von Frau Meyer in ihrer erzählerischen Normallage wieder, auch das Zitat

e hondheb fer $=n$ mehlsack $(19,10)$.

Ingrids Wiederholung der Formel ist relativ weit zum Standard verschoben:

e handhebe für=n mehlsack $(19,18 / 21)$.

Frau Zimmermann wiederholt ebenfalls standardnäher als in ihrer ersten Formulierung:

e handheb fer $=n(19,19)$.

Die Standardverschiebung zeigt vermutlich die Orientierung auf Ingrids Lernbemühungen und hat bestätigende Funktion. Frau Zimmermann bricht ab und leitet in den Erzählstrang zurück, indem sie in markiert dialektaler Lage (mit phonetischer Verschiebung und dumpferer Sprechweise) reformuliert. ${ }^{27}$ Sie fokussiert dabei die Verwendungsweise der Formel als dialektale Rede, zunächst durch topikalisierende Heraushebung des phonologisch entscheidenden Formulierungselementes

$<e$ hondheib $>$ hot se gsacht $(19,19-20,1)$

und dann durch die Wiederholung der gesamten Formel:

e hondheib fer $=n$ mehlsack ned $\uparrow(20,1)$.

Das Zitat der Beschwerde von Frau Hansen dagegen ist phonetisch standardnah, mit höherer und dünner Stimme und einer verwunderten Intonation gesprochen: ah sie habn misch ja auf dn arm genommen $(20,6)$. Insgesamt ist die Redeweise als „fein" gekennzeichnet.

Der Typ der formelhaften Sprechhandlung und ihre spezifische kontextuelle Bedeutung wird auf mehrfache Weise verdeutlicht, wobei erzählungsinterne und -externe Erklärungen miteinander kombiniert werden:

27 Zur Rolle der Sprachvariation für formelhaftes Sprechen vgl. z. B. Burger et al. (1983); dort wird der Transfer zwischen Hochdeutsch-Schweizerdeutsch und umgekehrt behandelt, wobei die interessantere Richtung die erste ist (vgl. S. 276f.). Einen Ansatzpunkt bietet auch das 'Transfer'-Konzept bei Auer (1982), das als Übernahme u.a. von formelhaften Wendungen definiert wird. 
(a) Die Formel ist zentraler Bestandteil der Handlung und wird an einem besonders interessanten und geglückten Verwendungsfall verdeutlicht; die Kerngruppenmitglieder zeigen durch ihr spontanes Lachen, daß sie die Formel und die mit ihr realisierte Sprechhandlung sofort verstehen.

(b) Für die „Novizin” Ingrid werden Formel und Sprechhandlung erklärt; und zwar durch:

- den Hinweis auf die Formelhaftigkeit und die lokale Spezifik: des is=n Monnemer ausdrugg $(19,11 / 16)$;

- die Angabe der entscheidenden Verwendungsbedingungen in Form einer generalisierten Darstellung des Anlasses (wonn jemond so neigierisch is; 20,3). Das ist eine auschnittweise Beschreibung einer Kommunikationssituation, die folgende Eigenschaften der Sprechhandlung erkennbar macht: Die Sprechhandlung reagiert auf eine negativ bewertete Bezugshandlung. Durch das intensivierende so in Verbindung mit der negativen Bewertungskomponente von neugierig wird die vorgängige Verletzung einer Regel des Sprechens, und zwar einer Distanzregel, als Anlaß für die Zurückweisung definiert.

- den Hinweis darauf, daß es sich um eine Unsinnsformel handelt, um eine Aussage über ein unmögliches Objekt (des gib=s doch ned des weeß ma; $19,20)$. Konstitutiv für die zurückweisende Reaktion ist hier also die Verletzung der Ernsthaftigkeitsbedingung. Diese Verletzung wird als klar erkennbar eingestuft (jedenfalls für "Leute wie uns"): des weeß ma.

Es geht hier also um das Kontern einer voraufgehenden Regelverletzung durch eine manifeste eigene Regelverletzung. Die Formelverwendung in der erzählten Situation wird durch die Betonung der Dialektalität für die standardsprechende Adressatin zusätzlich verrätselt. Verdeutlicht wird die Herstellung einer Kommunikationsbarriere.

(c) Auch Frau Hansen wird die Sprechhandlung erklärt: do is die hinnere un hod bei denne onnere gfrogt was des is $(20,2 / 4)$. Wie diese Erklärung ausgesehen hat, ist kaum erschließbar. Die zitierte Äußerung von Frau Hansen bei der zweiten Konfrontation sie habn misch ja auf dn arm genommen $(20,6)$ gibt das Ergebnis ihrer eigenen Interpretation des Ereignisses in ihren eigenen Sprechhandlungskategorien wieder. Diese Replik erscheint in besonderer Weise unangemessen aus der Sicht von Frau Zimmermann und der Zuhörerinnen: an dieser Stelle lachen sie zum zweiten Mal. Der besondere Erfolg für Frau Zimmermann und Frau Meyer liegt darin, daß Frau Hansen durch die verzögerte Reaktion zugeben muß, daß sie hereingefallen ist, weil sie das Rätsel alleine nicht lösen konnte; und weiter ist die Replik decouvrierend, weil diese Art der Thematisierung kein Kontern mit vergleichbaren Mitteln darstellt - der Replik fehlt jede Schlagfertigkeit; Frau Hansen wird damit als hilflose Verliererin präsentiert. Diese Typisierung entspricht genau einem im Kontext aus einem anderen AnlaB explizit ausgedrückten Urteil, wonach Frau Hansen nicht weiß, 
wie man sich verhalten soll - Frau Hansen wird als sozial inkompetent charakterisiert (vgl. auch den Beispieltext "Geschlossene Gesellschaft” - als leiderin weeß die ned emol wie se sisch vehalde soll; 7,1/2 - und die Analyse im vorigen Beitrag, Kap. 5.3.).

Die „Feinheit” ausdrückende Sprechweise des Zitats, und zwar eine etwas betuliche und pikierte "Feinheit”, symbolisiert Frau Hansen zugleich als Angehörige einer anderen Welt, in der Sprechhandlungen und Formeln der von Frau Zimmermann verwendeten Art nicht zur Normalform der Kommunikation gehören. Frau Hansen, die als Leiterin ihre Klientinnen steuern und kontrollieren will, kann die kulturelle Eigenständigkeit und die allergische Gegenwehr gegen Außensteuerung bei den Betroffenen nicht richtig einschätzen: Die Filsbachfrauen haben für Frau Hansen etwas Beunruhigendes.

(d) Die Erklärung von Frau Zimmermann gegenüber Frau Hansen verdeutlicht dreierlei:

- Einmal wird durch die Darstellung einer vergleichbaren Sprüchepraxis des Bruders (als von jemandem, der unzweifelhaft „einer von uns” ist) verdeut-

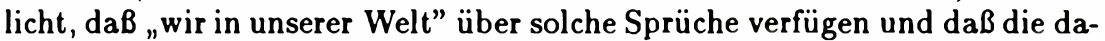
mit auszuführenden Sprechhandlungen zum normalen Repertoire gehören. Das ist das szenische Äquivalent zu der Charakterisierung als Monnemer ausdrugg gegenüber Ingrid.

- Zum anderen wird ein Bildungsmuster für derartige Sprüche demonstriert: "das ist ein $x$ für ein $y$ ", wobei $z$ wischen $x$ und $y$ nach der Weltkenntnis bzw. dem gesunden Menschenverstand keine sinnvolle attributive Relation hergestellt werden kann.

- Und schlieBlich kontrastiert der grobe Ausdruck gänsaasch mit der „Feinheit” der voraufgehenden Replik von Frau Hansen; hier wird noch einmal die soziale Distanz markiert.

Die Erklärung hat keinerlei Züge von Zurückweichen oder einem Versöhnungsangebot, sondern sie ist eine Demonstration der Stärke. Dieser Beitrag wird erneut mit Lachen quittiert.

(e) Aus AnlaB der Rückfrage von Ingrid gibt Frau Zimmermann eine plausibilisierende Hintergrundinformation, die wesentlich die Funktion der Realitätsbestätigung hat: Das ist die Praxis in "unserer Welt". Außerdem werden noch einmal die Verwendungsbedingungen durch die kondensierte Wiedergabe eines typischen Kontextes verdeutlicht: wo ma gsachd hod was gib=n des $\uparrow$ än riggel fer $=n$ gänsaasch; $(20,17 / 19)$.

Diese Unsinnssprüche werden sowohl gegenüber Außenstehenden als auch gegenüber Mitgliedern der eigenen Welt mit der beschriebenen Funktion der Zurückweisung verwendet. Außenstehenden gegenüber können sie auch gezielt als Rätsel zur Verunsicherung oder als Test (in der Art von Initiationsprozes- 
sen) verwendet werden (eine Funktion, die bei der Konfrontationserzählung vermutlich nicht vorliegt; das wäre der Leiterin gegenüber zu gewagt).

Eine milde Form der Zurückweisung durch einen Unsinnsspruch erfährt auch einmal Ingrid auf eine zu neugierige Frage: Sie fragt Frau Kunz, was sie während der Fastnachtstage abends unternimmt, und bekommt zur Antwort:

\section{$1 \mathrm{KU}$ : isch geh on de Neggaa ilvetritschle fange* \\ $\mathrm{K}:$ \#ZU IN \\ * *ZU fRAU X *}

\section{X: ja $\downarrow$ mer treffe uns uff de Neggaaries grasbischel dreizehn $\downarrow$}

Die Unsinnigkeit liegt hier im ersten Spruch (1) darin, daB ilwetritschle im ganzen pfälzischen Raum bekannte Phantasiewesen sind (das pfälzische Aquivalent zum bairischen Wolpertinger). In der Fortsetzung des Spruches (2) wird die Regelverletzung auch für Rezipienten ohne lokale oder regionale Kenntnisse eindeutig: grasbüschel dreizehn auf der Neckarwiese setzt die Zählung des sinnvollerweise nicht Zählbaren und zudem die Benutzung als geographisches Orientierungssystem voraus.

Ein Beispiel für die Zurückweisung eines Übergriffs aus der eigenen Gruppe ist die Verwendung von isch glaab dir brenn=de kiddel durch Frau Kunz einem anderen Gruppenmitglied, Maria, gegenüber. Zwischen beiden bestehen anhaltende Divergenzen hinsichtlich des sozialen Stils. Maria findet die Sprechweise von Frau Kunz ordinär und so rischdisch aus de Filsbach. Frau Kunz macht aber auch ihrerseits Maria „Vorschriften”. Darüber kommt es zu einem Wortwechsel:

1 MA: isch lob mir dehääm nix gfalle- glaabsch du donn vun dir $\uparrow$

2 MA: isch glaab=s gehd los

$3 \mathrm{KU}$ : $\quad$ isch glaab dir brenn=de kiddel

4 MA: sigsch des is vidder so e redensart $\downarrow$

Hier liegt ein anderes Konstruktionsmuster für Zurückweisungen vor, und zwar durch direkte Beschuldigung bzw. negative Qualifizierung des anderen (isch glaab dir brenn=de kiddel) oder der Situation, für die der andere verantwortlich gemacht wird (isch glaab=s gehd los). Die Konstruktionsform ist in beiden Fällen ähnlich: ich glaube + Hauptsatz, d.h. ohne Konjunktion daß und ohne Inversion. Das ist ein sehr verbreitetes Muster für Spruchbildung (vgl. ich glaub ich spinne, ich glaub dich sticht der Hafer, ich glaub dir gehts zu gut, oder scherzhafte Neubildungen wie ich glaub mein Pferd schielt). Bei dieser Verbindung von modalem Rahmensatz und Hauptsatz handelt es sich um eine spezifische Aussagemodalität, die nichts mit Abstufungen der Gewißheit über die Realität zu tun hat (wie es bei der Verwendung der Konjunktion daß der Fall wäre: ich glaube $d a \beta d u$ spinnst), sondern mit der Mißbilligung von etwas, was sein könnte, aber nicht sein soll bzw. vom Sprecher nicht geduldet würde. 
Bei diesem Beispiel zeigt sich ein weiterer Sequenztyp des formelhaften Sprechens. Hier geht es bei der Reaktion auf die jeweils voraufgehende formelhafte Beschuldigung um Steigerung und Übertrumpfen. Die Dynamik der sich abzeichnenden Entwicklung wird von Maria aufgehoben durch ihre ÄuBerung auf der Metaebene. Bei anderen Gelegenheiten, vor allem bei männlichen Sprechern, kann ein solcher Austausch von sich gegenseitig übertrumpfenden Beschuldigungen der Beginn eines handfesten Streits sein.

\section{Fazit: „Mach kä schbrisch”}

Bei unserer Untersuchung des formelhaften Sprechens haben wir uns darum bemüht, die Dynamik der Sprachverwendung zu erfassen. Dazu haben wir vier konstitutive Aspekte miteinander in Beziehung gebracht: Interaktionsmuster (wie Tratsch, Witzeerzählen, Problembehandlungen), Interaktionsmodalitäten beim Reden über die Welt, Formulierungsverfahren und die Gliederung des Repertoires an sprachlichen Formeln (selbstgebildete und vorgeprägte Formeln unterschiedlicher Herkunft).

Die Untersuchung gibt Einblick darin, wie die soziale Bedeutung von Sprache situativ, in der Sprachverwendung erlebt und geprägt wird. Ein wichtiger Faktor dabei ist, da $B$ die Sprachverwendung die Relevanz unterschiedlicher Wissensbestände für die Interpretation und die erfolgreiche, von den anderen honorierte Beteiligung signalisiert. In unseren Analysen ging es dabei um unterschiedliche Wissensbereiche wie die unmittelbare Erfahrung und ihre Verarbeitung in Geschichten, das Wissen über die Lebensbedingungen und die Normalitätsvorstellungen in einer bestimmten, lokal verankerten sozialen Welt und Wissensbestände, welche diese Welt transzendierende Normen betreffen. Die Formelhaftigkeit des Sprechens fungiert als Kontextualisierungshinweis auf den Thematisierungs- und TypisierungsprozeB in der Gruppe. In diesen wiederum geht das historische und kulturelle Hintergrundwissen ein.

In dieser Hinsicht zeigt die Analyse spezifische Eigenschaften der untersuchten sozialen Welt, und zwar die Konkretheit und Realitätsbindung auch des spielerischen formelhaften Sprechens. Die formelhaften Formulierungen verweisen auf die konkret erfahrene eigene Welt. Und ihre Verwendung ist an spezifische Kriterien der Angemessenheit gebunden: Sie müssen in Übereinstimmung mit dem konkreten Weltwissen und in diesem Sinne spezifisch verwendet werden (es genügt nicht, daß die Formulierungen einfach nur markant und lustig sind, wenn sie weltspezifische Kontexte indizieren). Die dargestellte Technik des formelhaften Sprechens ist für Externe schwerer zu erlernen als z.B. das Erzählen von Witzen und das spontane Formulieren von witzigen Bemerkungen im Einklang mit den kulturspezifischen Vorstellungen von Komik. Insbesondere das spezifisch geprägte formelhafte Sprechen gehört zu den Verfahren der Symbolisierung sozialer Identität, mit denen die Sprecher zusammen mit der Verdeutlichung einer Weltsicht und eines sozialen Stils die Zugehörigkeit zu einer bestimmten sozialen Welt ausdrücken. 
Die Analyse gibt also zusammen mit den allgemeinen Einsichten immer auch Einblick in die spezifische Mannheimer, genauer innenstädtische Sprachkultur. Mit den aufgezeigten Verfahren läßt sich genauer rekonstruieren, worin die sprachlichen Besonderheiten der „Mannemer Gosch” bestehen, für die ja die Präferenz für formelhaftes Sprechen einer bestimmten Art als charakteristisch angesehen wird - wobei Vergleiche zeigen müssen, wie exklusiv diese Eigenheit eigentlich ist bzw. was daran exklusiv mannheimerisch oder auch regional ist.

Die dargestellten Beobachtungen belegen vor allem die Vorliebe für das relativ unauffällige formelhafte Sprechen, z.B. mit selbstgebildeten Charakterisierungen und Maximen. Das Bild ist zumindest um zwei weitere Erscheinungsweisen zu ergänzen:

- das Sprüche-klopfen (schbrisch klobbe), d.h. das gehäufte Verwenden von festen Redewendungen (das ansatzweise bei den Unsinnsformeln sichtbar wird);

- die angeberische Rede, d.h. eine - gemessen mit den Kriterien der lokalen Welt - überzogene Form der Selbstdarstellung, die als Reaktion die Formel provoziert:

mach kä schbrisch.

\section{Beispieltext: „Der arme Otto”}

S. 1

1 ZI: (...) un no hod se noch drei * ä:h * ä:h ki"rsch/ nix

2 ZI: kirsch * vassa bschdelld un so * >na havg=isch gsa vie

$3 \mathrm{ZI}: \mathrm{geh}=\mathrm{da}=8 \uparrow$ seschd se jo woin alde (...)

$4 \mathrm{SU}$ :

5 MÜ: >hausdrache

6 IN : ihrn mann

$7 \mathrm{ZI}$ :

8 MÜ:

9 IN: is=er $\uparrow$ än hau"sdrache $\uparrow$ $\rightarrow$ jajal $(\ldots \ldots)$

$10 \mathrm{ZI}$ : der hod alles schaffe misse $\downarrow$ ohje ohje $\downarrow$

11 MÜ: ja

12 IN : ehemann $\uparrow j a$

$13 \mathrm{ZI}$ :

$14 \mathrm{kU}$ :

15 IN: des die fraa die do in H5 vohnt $\uparrow$

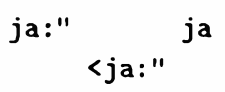


$16 \mathrm{ZI}$ :

ja:

$17 \mathrm{KU}$ : ja: $\downarrow$ die wu do $\mathrm{ihr} / \mathrm{ihr} / \mathrm{ihr} / \mathrm{ihr}$ brilljonde do

18 IN : filn $\uparrow$ äh/

$\mathbf{K}:$

\section{LACHEN}

$19 \mathrm{KU}: \underline{(\ldots)}$

$20 \mathrm{SU}$ : $\quad$ die

21 IN : aaah $>$ des is die do $\uparrow$ un die heiBt Gerddl $\downarrow$

22 2I:

Ge"rda

$\mathbf{R}$ :

GEDEHIT

23 SU: brü"lljande

$24 \mathrm{KU}$ :

ja: Gerdda

25 แH:

der

26 IN :

sun die heibt Gerddl

S. 2

$1 \mathrm{ZI}$ Gerda

2 HH: krigg=de eisschrongg abgschlosse daB=a ned zuviel friBd $\downarrow$

3 mü:

Gerda

4 2I: $\quad(\ldots)$

5 SU: LACHT ERSTAUNT

$6 \mathrm{kU}: \quad(\ldots)$

7 НH: $\quad \rightarrow$ (oh daB=a ned soviel friBd)

8 MÜ: ja

9 2I :

$10 \mathrm{SU}$ :

*aach"

$\mathbf{K}$ :

\#ERSTAUNT\#

$11 \mathrm{HH}: \leftarrow$ der kriggd de eisschrongs abgschlossel

12 ZI: kriggd=n abgschlosse $\downarrow$ ja un/

13 HH: jaja taja * un venn=a brav is

14 MÜ: $\quad$ ia:"

15 HH: kriggd=a haløi dafel schoklad $\downarrow$ ve"he venn=a mehr vill

16 MÜ:

$\mathbf{K}$ :

DURCHEIMANDER

17 ZI : ־Un alle schdund ei"ne zigaredd!

$18 \mathrm{HE}$ : jaja

19 HH:

isch hab=a ima paar

20 IN :

pa:s $\uparrow$ 
$21 \mathrm{WH}:$ gevee ach gott der=s arbeidslos $\downarrow$

22 rü: $\quad$ der arbeided nix

$23 \mathrm{KR}$ :

24 IN :

arbeitet der $\frac{\text { des }}{\text { nix }}$

$25 \mathrm{ZI}$ :

$\rightarrow$ nö nö der is de ganze dach (...)

26 แH:

sisch hab gsachd ger du sau/ du

27 RR: läß=der sich gfallen $\uparrow$

$28 \mathrm{ZI}: \underline{(\ldots)}$

29 HH: saufsch avva aa fünf verddl voi $\rightarrow$ varum soll der bloß ää

$30 \mathrm{KU}$ :

mir vare mol/ * mir

31 HH: zigaredd raache in de schdunnd $\uparrow$ LACHT

$32 \mathrm{KU}$ : vare mol fordd >var die * fra Kaddovitz debei * un do hod er

S. 3

$1 \mathrm{ZI}$ :

$(\ldots \ldots)$

$2 \mathrm{KU}$ : sisch vas beschde"lle volle $\uparrow$ do"/ vas veeß isch $\uparrow$ $\mathrm{K}$ :

*<a:s" $\uparrow$

IKEIFEND

3 BO:

isch

$4 \mathrm{ZI}$ :

der derf doch $\underline{\text { nixl }}$

$5 \mathrm{KU}: \underline{\text { hodse } \uparrow}$

घa"s $\rightarrow$ hod se gsacht $\uparrow *$ isch glaab

$\mathbf{K}:$

$6 \mathrm{BO}$ : var debei

$7 \mathrm{KU}$ : daß du verriggd bisch $\uparrow$ do geh hääm un mach doi (...)* avva K :

KEIFEND:

8 2I:

$9 \mathrm{KU}$ : sie" hod aøva gfresse =s deierschde

10 MÜ:

o0000h $\leftarrow$ du:" kriggsch/ du

$11 \mathrm{HE}$ :

$12 \mathrm{KU}$ :

13 мü: kriggsch äh:/

14 WH:

hagg/ nää: eier

eier- * eier

$15 \mathrm{KU}$ :

$\rightarrow$ domols hod=a haggbrode griggd des va=s

$16 \mathrm{HH}$ : mid schingge

$17 \mathrm{KU}$ : billigschde bloß fünf maag gekoschd

18 WH :

eier mid schingge

un die

$19 \mathrm{KU}$ : onner hod was gfresse des/ des hod fuchzehne gekosch $\downarrow$ *

$20 \mathrm{WH}$ : griggd=a meischdens | 
$21 \mathrm{SU}: \geq \mathrm{aach}<$

$22 \mathrm{KU}:$ Kra"s hod se gsachd venn da=s ni baßd do gehsch hääm un

$K$ : *SCHARF, HOCH

23 MÜ:

>ouu<

24 IN :

LACHT

25 2I :

$26 \mathrm{KU}:$ kochsch da rast $*$ ooouuu

(isch glaab)

R: SCHARF, HOCH

27 HH: $\quad$ Sschri"me ivoer de ozea:n-

28 IN : LACHT

S. 4

$1 \mathrm{ZI}:$ ja ja $(\ldots \ldots)$ nänä

2 HE: var der bra:v *

$3 \mathrm{KU}:$ der hod schä: Resse

4 WH: schvi"me ivver de ozean $\downarrow$ *

R : DURCHEIMANDER

5 แH:

derss doch vun Ameriga- schvi"mon ivoer

6 MÜ: >do hod=a=s (besser)

$(\ldots \ldots \ldots \ldots)$
7 HE:
ach der volld/
Sder voll $=$ doch

$8 \mathrm{KU}$ :

die hod=n schä zommegschisse $\uparrow$

9 HH: de ozean $\downarrow$ LACHT

nänä

$10 \mathrm{ZI}$ :

sach/ hot sexn ne

$11 \mathrm{HE}$ : nivverfahre vu soin vadder gschdorve is nidf

$12 \mathrm{ZI}$ : geloBt nä näl

$13 \mathrm{HE}:$ die hod=n ni"schd geloßd $\downarrow$

14 WH:

>der gär vielleisch

$\mathrm{K}$ :

*<der vär nimmer

*HOCH

$15 \mathrm{KR}$ :

$\underline{\mathrm{a}^{\prime \prime} \mathrm{ch}}$

$16 \mathrm{ZI}$ : ah der pär nimmer kumme $\downarrow$

$17 \mathrm{HE}$ : garnimmer kummel de"r vär nimmer kumel

$18 \mathrm{HH}$ : ku"me†"

$\mathbf{K}$ :

19 MÜ :

der gär nimmer

$20 \mathrm{ZI}$ :

21 mÜ: kume $\downarrow$

$22 \mathrm{ME}$ :

achia: 
$23 \mathrm{ZI}:$ mu=8: 80" genach denm gherd=s ned onnerschd I

$24 \mathrm{KU}$ :

\section{e:ve:}

25 แH:

ah des is klar

26 MÜ:

ha jal

$27 \mathrm{ZI}$ :

$28 \mathrm{KU}$ :

29 MÜ:

den Rherd=8 doch

ned annerschd

do havve ner kä

30 IN : LACHT

S. 5

$1 \mathrm{ZI}:$ nei:n† $\underline{\mathrm{nix}}$ nix

$2 \mathrm{SU}$ :

aaa

$3 \mathrm{KU}$ : bedengge $\downarrow$

kä bedauern

$4 \mathrm{KR}$ :

5 IN:

un vie soll wa den etz so/

$6 \mathrm{ZI}$ :

bei ihr hod=a kei"ne zigaredd rauche

$7 \mathrm{KU}$ : saio vei=a beglobbd is: $\uparrow$

$8 \mathrm{KR}:$ sisch des gfalle $\uparrow$

9 ZI: dürfe $\downarrow$ muBd=a nau"s * uff=s * trebbehaus $\downarrow$ * venn 10 MÜ:

11 ZI : avga bsu"ch do var $\downarrow$ die" havge raache

12 MÜ: oooh

$13 \mathrm{ZI}$ : kenne LACHT

$14 \mathrm{HE}$ : un un der ihr mudda vo se noch

15 WH: LACHT do hädd=isch arva vas gsacht |

$16 \mathrm{HE}:$ gelebd hod $\uparrow$ die var noch

$17 \mathrm{HH}$ : die var genauso miserabel gell $\uparrow$

18 HE: schlimma: $\uparrow$ un die hod vun ihrm/ vun demm soim

19 MÜ: noch schlinma var noch/

20 HE: geld gelebd venn er ovends noch vas esse gevolld hod hod=a

$21 \mathrm{HE}: \mathrm{ni}$ "schds mehr griggd

22 MÜ:

on on fernseh:

23 XR: ja jal

24 LM: ah ja

$25 \mathrm{KR}:$ für=n $\operatorname{mann} \uparrow(\ldots \ldots \ldots \ldots)$

26 IN : vas is=en des für=n mann $\uparrow$ frag ich mich 
$27 \mathrm{ZI}: \quad \geq(\ldots \ldots \ldots \ldots \ldots)$

$28 \mathrm{HE}:$ des: is=n debb hear $\uparrow$

$29 \mathrm{KU}:$ ah ia des: en de"bb

$30 \mathrm{HH}$ :

der hod/ der/ der hod vun Vietnam ä

31 IH: auch |

S. 6

1 UH: bissel vas ghabd ääh-

$2 \mathrm{ME}:$

3 IN :

ach des is=n solda: $d \uparrow$

4 Нн: $\quad \mathrm{ja} \downarrow$ der var/ der var/ (...)

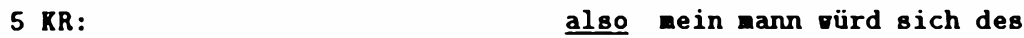

$6 \mathrm{HE}:$ gsacht

$7 \mathrm{KU}: \quad$ ach godd ah $*$ ach godd ach godd ach godd

$8 \mathrm{KR}:$ nit gfalln $\underline{\text { lassn }}$

9 IN :

ach der

$10 \mathrm{ZI}$ :

$11 \mathrm{KU}$ :

ahia

$12 \mathrm{HH}:$

der

13 IN: is virklich ä bissel bscheu/ also-

$14 \mathrm{KU}$ : hod=n schdisch ins griene $\downarrow$

15 HH:

avra vem=ma mid=m schbrischd

16 IN : ach sol

$17 \mathrm{ZI}$ : ahia der hod vas $\downarrow$

$18 \mathrm{HH}:$ ned $\downarrow$

nänäl

oh der

$19 \mathrm{KR}:$

20 IN :

also eigentlisch net $\downarrow \underline{i a \uparrow}$

nänä

$21 \mathrm{ZI}$ : schbrischd avea ned viel

$22 \mathrm{KU}$ :

23 WH:

nää nää

24 HE:

Sja: avva venn/ avva venn=a aus

25 WH: sisch raus gehd * der schbrischd sehr nedd und äh-

26 ZI:

$27 \mathrm{KU}:$

28 MÜ: jaja venn=a aus/

ja

29 KR: ja 
30 2I: net (...)

31 HE:

32 WH: venn sie ned zuherd $\uparrow$

33 MÜ:

oh ja nä nä

$\mathbf{R}$ :

DURCHEIMANDER

S. 7

1 WH: der/ venn sie ned zuherd $\mid$

$2 \mathrm{KR}$ :

$\mathbf{K}$ :

ja ja ja

$$
\text { oh ja na na }
$$

3 ZI:

4 MÜ:

ja $\downarrow$ ja $\downarrow$ vir han

$5 \mathrm{KR}$ : ja ihn inner alleine hier gehabt $\downarrow$ da auf=m platz (...)

6 ZI: gege denn is nix zu sachel

$7 \mathrm{HE}:$

8 мÜ:

9 IN :

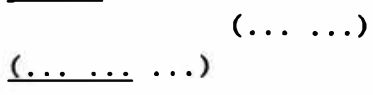

ja un vo hat die

$10 \mathrm{ZI}$ :

ahjo

11 IN : den kennegelernt $\uparrow$ hier $\uparrow \quad$ var der/ var der soldad hier $\uparrow$

$12 \mathrm{ZI}:(\ldots \ldots)$

$13 \mathrm{SU}:$

L.ACHT

14 mü: $\Rightarrow$ do var=er bschdinmd vollgsoffe vie=a die kennegelernd hod

$15 \mathrm{ZI}$ : der var soldad $\downarrow$ un var in Korea * nit $\uparrow$ de"r un veil er

16 IN : der ahia aaaahtahal

$17 \mathrm{ZI}$ : dreißisch jahr bei:n $>(\ldots)$
$18 \mathrm{hÜ:} \quad$ vie seschd ma $\uparrow$ mir sache * beim

$19 \mathrm{ZI}$ : bund beim milidär par * is er jo donn endlasse vorre nit $\uparrow$

$20 \mathrm{KU}:$

21 IN :

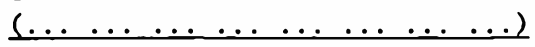

22 WH: <der hot se avva mol schä bschi"sse gell $\uparrow$ des hedd se aa ned $23 \mathrm{ZI}$ : ja $\uparrow$ vege vas $\uparrow$

24 HH: gedengd LACHT GEHÄSSIG ja ja ah nõ: * der hod

25 ZI : ah do:ch $\downarrow$ der hod

26 HH: ima geld vo sie nix devun gevißd hod $\downarrow$

27 MÜ:

do hod se

$28 \mathrm{ZI}$ : sie/ i"sch var schunn debei vo=a=8 geld hod un hod=s=ere

30 แH:

31 Mü: doch ihr finger druff

Sia arva 
S. 8

121 : hiereleggd l un no hod=a/

2 HH: die hodd * der hod enol vas ghold * (...) do hod=a

3 II: Sun donn äh $\rightarrow$ hod se ssacht ach Otto isch bin

4 SU: $\quad \operatorname{LACHT}(\ldots \ldots) \quad(\ldots \ldots \ldots$

5 Hн: beschissn

6 ZI: in dej bank grad änol noił seschd se isch hab gnäänd • isch

7 sU: $(\ldots)$

$8 \mathrm{ZI}$ : hedd des schunn des ge"ld net $\uparrow$ der hod=s in do"llar

9 ZI: kriggt * no hod se die dollars gschbard LACHT hod se ä

$10 \mathrm{ZI}$ : kischd voll dollar ghabd un no hod se gsacht isch

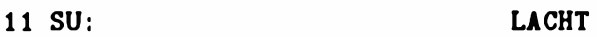

12 ZI : vardd $\uparrow$ * do vare se zvee naak * dreiBisch * zvee naak

$13 \mathrm{ZI}: *(\ldots . .$.$) dreiezvonzisch \rightarrow \rightarrow$ seschd se isch vardd die

R: GERAUNE IM hINTERGRUND/ BEMUNDER DIE BASTELARBEIT,

14 ZI: kume vidda uff zvee maak fuchzisch

$\mathrm{R}$ : BESONDERS UH

15 แH:

$16 \mathrm{KR}$ :

oh des is schö:n】

$\mathbf{K}$ :

sehr schön:

* JEMAND LACHT*

17 ZI : ooh $\uparrow$

$18 \mathrm{HE}$ : hear ** frieher hod na fer/fer zäh dollar ivver

19 HE: verzisch äh maag griggd (.....)

20 NA :

ia vie isch=8 erschde

$21 \mathrm{ZI}$ :

vas dengre sie $\uparrow$

22 HE:

isch veeß $\uparrow$ vonn isch als no

23 NA: mal nach Amerika bin isch hab sivje maak un zoonzisch

$\mathrm{R}$ : DURCHEIMANDER

$24 \mathrm{ZI}$ : vie die denn $(\ldots \ldots \ldots)$ do var de dollar noch*

25 MA: pfennisch fer ä"n dollar (...) un heid noch zvei naak

S. 9

1 2I: vier naak zronzisch do hozdie õgerge kenne

2 HE: vier laak żanzisch

3 NA: sechzisch (..) kriggd ghabt (..)

R: $\quad$ GEMURHEL 
4 ZI: net $\uparrow$ dollar $\downarrow$ un domols var=s noch billisch net $\uparrow$

5 HE:

ja isch

6 MÜ:

$\mathrm{K}$ : DURCHEINAKDER

ja ja

7 HE: hab=8 aa kriggd $\downarrow$ isch bin reisch isch hab aa vidda en haufe

8 HE: kriggd $\downarrow$ än haufe

$9 \mathrm{KU}: \quad \leftarrow<a ̈ n$ haufe pfui * is unanschdändisch $\downarrow$

$10 \mathrm{HE}:(.$.

$11 \mathrm{KU}: \rightrightarrows$ schigge die de haufe vun ameriga do rivver/ isch dongg

12 HE: LACHT

$13 \mathrm{KU}$ : schä: $\downarrow$ * der muß schä gschdungge havze bis er do hivve

14 WH: do ded isch $m i s c h$ avga vervö"hne * >un ded ned immer fer die

$15 \mathrm{SU}$ :

var doch in der kühl/

$16 \mathrm{KU}:$ घar| LACHT

17 WH: onnere mensch $\downarrow$ * Lodda die katze kriggn jetz: dann extra $18 \mathrm{SU}$ : kühltruhe |

19 ZI:

un un eh:

ja un eh

20 HE:

$21 \mathrm{WH}$ : noch e pund rindfleesch

LACHT

22 ZI : <fra Wi"chmer vas verddrn der zur silverhochzeit zu fresse

$23 \mathrm{ZI}: \mathrm{krigge} \uparrow$

24 WH: hoffenl/ ah hoffenlisch kriggd=a ä runschdek

25 Мн: un kä/ kä * un kää * eier nid schingge $\downarrow$

26 IN :

LACHT die katz odda

27 ZI: \#de Oddo $\underline{\text { die hod/ nä }}$

$\mathrm{K}:$ LACHEND*

28 HH: de Oddo $\downarrow$

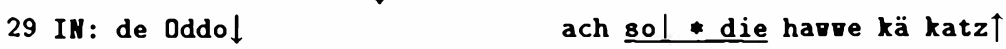

S. 10

1 ZI : nä

2 HE:

derre geng $=8$ schleschd derre

3 WH: <ach godd die konn doch de"nn ned leide un donn ä

$4 \mathrm{HE}: \underline{\operatorname{ratz} \uparrow}$

$5 \mathrm{kU}: \quad$ ja ja*

$\mathrm{K}:$ :LACHEND*

6 แH: katzl

$\mathrm{K}$ :

THEMENHECHSEL: FRISEUR / KATZEN 